\title{
Mechanisms of Enzalutamide Resistance in Castration-Resistant Prostate Cancer and Therapeutic Strategies to Overcome It
}

\author{
Yuanyuan Wang ${ }^{1}$, Jiyuan Chen ${ }^{1}$, Zhengjie $\mathrm{Wu}^{2}$, Weihong Ding ${ }^{3}$, Shen Gao ${ }^{2}$, Yuan Gao ${ }^{4}$, \\ and Chuanliang $\mathrm{Xu}^{2}$ \\ ${ }^{1}$ Fudan University \\ ${ }^{2}$ Changhai Hospital \\ ${ }^{3}$ Huashan Hospital Fudan University \\ ${ }^{4}$ Fudan University School of Pharmacy
}

July 27, 2020

\begin{abstract}
Prostate cancer (PCa) is the second most common malignancy in men, and androgen deprivation therapy (ADT) is the first-line therapy. However, most cases will eventually develop into castration-resistant prostate cancer (CRPC) after ADT treatment. Enzalutamide (Enz) is a second-generation androgen receptor inhibitor approved by the Food and Drug Administration to treat patients with CRPC. Unfortunately, patients receiving Enz treatment will ultimately develop resistance via various complicated mechanisms. In this review, we introduce the emerging information on resistance mechanisms, including androgen receptorrelated signalling pathways, glucocorticoid receptor-related pathways, and metabolic mechanisms. Notably, lineage plasticity and phenotype switching, gene polymorphisms, and the relationship between microRNAs and drug resistance are addressed. Furthermore, potential therapeutic strategies for Enz-resistant CRPC treatment are suggested, which can help in the discovery of more effective and specific regimens to overcome Enz resistance.
\end{abstract}

\section{Introduction}

Prostate cancer $(\mathrm{PCa})$ is the second most common malignancy in men and the fifth leading cause of death worldwide (Rawla, 2019). Patients with early-stage prostate cancer have a good prognosis (Trewartha and Carter, 2013). However, advanced prostate cancer is associated with high mortality with the development of invasion and metastasis (Zong and Goldstein, 2013). Since Huggins and Hodges discovered that PCa responds to androgen therapy ( $R$, 2002), androgen-deprivation therapy (ADT) has become the first-line treatment use to suppress PCa progression. Despite an initial therapeutic efficiency of 80-90\% (Trewartha and Carter, 2013), nearly all patients treated with ADT will ultimately develop castration-resistant prostate cancer (CRPC) (Davies, Conteduca et al., 2019).

Patients with CRPC show a sustained increase in serum prostate-specific antigen (PSA) levels after castration treatment (Saad, Chi et al., 2015), which is generally attributed to the reaction of the androgen receptor (AR), which may be the result of AR gene amplification or other mechanisms (Cai and Balk, 2011, Tran, Ouk et al., 2009). The androgen biosynthesis inhibitor abiraterone and AR antagonist enzalutamide (Enz) are two novel agents targeting androgen action to treat patients with CRPC and were approved by the Food and Drug Administration (FDA) in 2012 (Nelson and Yegnasubramanian, 2013). Enz is a potent AR signalling inhibitor without agonistic activity. It suppresses the nuclear translocation of activated AR to prevent its localization to androgen response elements and coactivator recruitment, which induces apoptosis 
while inhibiting proliferation of the CRPC cells (Erdogan, 2018 , Jiang, Chen et al., 2020). A phase III clinical trial reported that patients treated with Enz survived months longer than those in the placebo group (Niu, Guo et al., 2018). Despite the initial therapeutic effect of Enz, CRPC patients will eventually become resistant to it (Erdogan, 2018). Therefore $>$ sout, it is urgent to explore new strategies to resolve resistance to Enz.

In this review, we summarized the emerging information on Enz-resistance mechanisms in CRPC, including AR-related signalling pathways, lineage plasticity, cytokines dysregulation, and gene polymorphisms. Furthermore, new potential therapeutic strategies to treat resistant CRPC which are promising for better therapeutic prognoses are introduced. We hope that these recently discovered mechanisms and emerging solutions provide some inspiration for the treatment of Enz-resistant CRPC.

\section{Mechanisms ofEnz resistance in CRPC}

\section{Restoration ofa ndrogen receptor signalling}

Androgen receptor reactivation

AR belongs to the steroid hormone receptor family and functions as a transcription factor. It contains three different functional domains: the N-terminal domain (NTD), the DNA-binding domain (DBD), and the carboxy-terminal ligand-binding domain (LBD). The main role of AR is to regulate genes encoding protein transcription needed for prostate function and the promotion of cellular differentiation in the normal prostate (Wong, Ferraldeschi et al., 2014).

Recently, several studies have demonstrated that AR is highly expressed and transcriptionally active in CRPC despite Enz treatment (Yuan, Cai et al., 2014). David Y. et al. (Takeda, Spisák et al., 2018) utilized an integrative analysis of ChIP-seq in primary specimens and identified a somatically acquired AR enhancer located $650 \mathrm{~kb}$ centromeric to the AR, which is frequently amplified in CRPC. Under low androgen conditions, additional copies inserted into this region are sufficient to increase cell proliferation and reduce cell sensitivity to Enz. Targeting this AR enhancer may become a potential method for treating resistant CRPC. Galectin-3, a member of the animal lectin family, significantly inhibited the therapeutic effect of Enz by increasing the expression of several AR target genes, such as kallikrein-related peptidase 3 (KLK3) and transmembrane protease serine 2 (TMPRSS2). Such enhancement of AR transcriptional activity and expression of AR-related genes leads to cell resistance to Enz (Dondoo, Fukumori et al., 2017). In contrast to its direct influence on AR expression, BMI1, a polycomb group protein, binds the androgen receptor, preventing ubiquitin E3 ligase-mediated AR protein degradation, which results in sustained AR signalling in CRPC cells (Zhu, Zhao et al., 2018). By constructing an Enz-resistant xenograft model, the authors found that the BMI1 inhibitor PTC209 significantly decreased Enz-resistant CRPC tumour growth. Thus, BMI1 is a novel therapeutic target for CRPC.

Recently, Hwang et al. (Hwang, Seo et al., 2019) indicated that resistant prostate cancer overexpressed or amplified cAMP-response element-binding protein 5 (CREB5), mediating the enhancement of AR activity at a subset of promoters and enhancers, including Myc and genes involved in the cell cycle. Notably, the pioneering factor forkhead box protein A1 (FOXA1) was also bound to CREB5/AR-binding sites and was necessary for CREB5-mediated resistance, which was not eliminated by Enz. The expression of the protooncogene c-Myc is positively associated with AR expression (Bai, Cao et al., 2019). Alex Bainbridge et al. (Bainbridge, Walker et al., 2020) observed the significant inhibition of AR-regulated gene expression upon siRNA-mediated I-kappa-B kinase (IKBKE) depletion or pharmacological inhibition of IKBKE in an LNCaPderived cell line resistant to Enz. Further analysis showed that IKBKE regulated AR levelsvia Hippo pathway inhibition to reduce c-Myc levels at cis-regulatory elements within the AR gene, leading to the formation of a large pool of AR. Another study showed that erythropoietin-producing human hepatocellular (Eph) receptors positively regulated AR by inducing proto-oncogene c-Myc expression, which was critical for Enz resistance. To date, several EphB4 inhibitors have been tested in clinical trials (Li, Lanman et al., 2020). Considering the low AR conditions after AR inhibitor treatments, Liu et al. discovered that deficient AR altered the balance between eukaryotic initiation factor 4E-binding protein 1(4EBP1) and eukaryotic translation initiation factor 
4F (eIF4F) complex formation (Liu, Horn et al., 2019). The loss of AR increased eIF4F assembly to drive the translation of a network of pro-proliferation mRNAs that were needed for tumour growth. Thus, the suppressing eIF4F may be a possible strategy for treating Enz-resistant CRPC.

Moreover, inhibitors of signal transducer and activator of transcription 3 (STAT3) and transactivation activity, namely, galiellalactone, (GPA500) (Thaper, Vahid et al., 2018), and triptolide (Han, Huang et al., 2017), have been proven to overcome drug resistance by targeting AR signalling. Tetra-aryl cyclobutanes are novel AR antagonists that are structurally different from Enz and inhibit the growth of Enz-resistant xenograft tumours (Pollock, Wardell et al., 2016). Proteolysis-targeting chimaeras (PROTACs) are used in an emerging approach that has recently attracted considerable attention. They are small molecules that control intracellular protein levels by recruiting targeted proteins into the ubiquitin-proteasome system (UPS) for selective degradation (Toure and Crews, 2016). Jemilat Salami et al. (Salami, Alabi et al., 2018) performed a head-to-head comparison of Enz and the PROTAC derivative ARCC-4 by utilizing different cellular models of PCa drug resistance. The results indicated that ARCC-4 was better at overcoming resistance in CRPC cellular models. Furthermore, at low nanomolar concentrations ARCC-4 inhibited the proliferation of ARamplified PCa cells. Hence, PROTAC-mediated AR degradation is a very promising method of Enz-resistant CRPC therapy. In addition to protein degradation by PROTACs, the orally bioavailable AR degrader UT-34 and interferon regulatory factor 8 (IRF8)-induced drugs are alternative strategies for Enz-resistant CRPC treatment (Ponnusamy, He et al., 2019 , Wu, You et al., 2020).

Androgen receptor splice variants

AR is composed of NTD, DBD, and LBD. When LBD binds to a ligand, the conformational changes of LBD allow AR to be transported into the nucleus where it binds to DNA. AR can undergo alternative splicing to generate isoforms that contain exons 1 to 3 with a deleted LBD. A truncated AR protein without the LBD fails to respond to AR targeting by Enz (J, Bubley et al., 2017).

AR variant 7 (AR-V7) is the most common variant that detected in circulating tumour cells from patients with CRPC, and it is significantly increased upon Enz therapy (Antonarakis, Lu et al., 2014). The splicing factor hnRNPA1 and the long non-coding RNA (lncRNA) Malat1 have been identified as contributors to Enz resistance by promoting the generation and expression of AR-V7 (Stone, 2017). Furthermore, it has been reported that tyrosine kinase ACK1 (TNK2) phosphorylates histone H4 functioned as an epigenetic modifier mediatingc a feedforward ACK1/pY88-H4/WDR5/MLL2/AR epigenetic circuit. The transcription of AR and AR-V7 was increased, leading to Enz resistance (Mahajan, Malla et al., 2017). Recently, Zhao et al. (Zhao, Peacock et al., 2019) found that arginine vasopressin receptor 1a (AVPR1A) was the most downregulated gene after the depletion of AR-V7 or the versatile coactivator VAV3. Therefore, suppressing AVPR1A in CRPC cells can decrease cell proliferation. Utilizing AVPR1A as a therapeutic target is effective not only for CRPC but also for bone-metastatic CRPC. High AR/AR-V7 expression and a positive correlation of AR-V7 with an E2F score indicated a supraphysiological testosterone (SPT) response (Lam, Nguyen et al., 2020). SPT produced an enduring response as shown by the sustained inhibition of AR-V7, E2F transcriptional output, and the DNA damage response (DDR) transcriptome. Hence, SPT treatment is a potent candidate for treating Enz-resistant CRPC. Moreover, CRPC cells can recruit more natural killer (NK) cells than normal prostate epithelial cells in the PCa microenvironment. NK cells altered microRNA34 and microRNA-449 to suppress AR-V7 expression and AR-V7-induced enhancer of zeste homologue 2 (EZH2) expression. Utilizing these immune cells provides a novel angle for approaching Enz resistance (Lin, Chou et al., 2017).

Protein homeostasis is another potential mechanism leading to drug resistance. Here, Liu et al. (Liu, Lou et al., 2018) demonstrated that UPS was inhibited in Enz-resistant prostate cancer. The interaction between heat shock protein family member HSP70 and functional E3 ubiquitin ligase STUB1 was required for AR/AR-V7 proteostasis. Suppressing HSP70 significantly inhibited tumour growth and resistance to Enz by decreasing AR-V7 expression. Similarly, aldo-keto reductase family 1 member C3 (AKR1C3) enhanced AR-V7 protein stability viathe alteration of the UPS. AKR1C3-targeted indomethacin greatly reduced AR/AR-V7 protein expression in vitro and in vivo through the activation of the ubiquitin-mediated proteaso- 
me pathway (Liu, Yang et al., 2019). A preclinical study indicated that the anti-apoptotic B cell lymphoma-2 (BCL2) protein inhibitor ABT263 increased ROS levels, leading to the inhibition of ubiquitin specific protease 26 (USP26) activity. As a result, the ubiquitination and ubiquitin-proteasome-dependent degradation of AR and AR-V7 were increased (Xu, Sun et al., 2020). Other agents, including fatty acid synthase inhibitor (FASN) IPI-9119, luteolin, triterpenoid antioxidant drug bardoxolone-methyl (CDDO-Me), and GnRH antagonist degarelix (Firmagon), have also been identified as inhibitors of the AR-V7 protein in Enz-resistant CRPC (Zadra, Ribeiro et al., 2019 , Naiki-Ito, Naiki et al., 2019 , Khurana, Chandra et al., 2020 , Cucchiara, Yang et al., 2019).

In prostate cancer cells, c-Myc is one of the most overexpressed genes and is frequently upregulated in CRPC. c-Myc promotes AR gene transcription and enhances the stability of full-length AR (AR-FL) and AR-V proteins without altering AR RNA splicing. The inhibition of c-Myc, such as with 5-Lox inhibitor MK591/quiflapon, can interrupt oncogenic c-Myc signalling to kill Enz-resistant cells (Bai, Cao et al., 2019 , Monga, Subramani et al., 2020). GATA2 is also a critical regulator of AR-Vs, and its cistromes considerably overlap with bromodomain and extra-terminal (BET) proteins (Chaytor, Simcock et al., 2019). Smallmolecule inhibitors of GATA2 are potent agents that suppress the expression and transcriptional function of both AR-FL and AR-Vs (He, Lanz et al., 2014). Moreover, the selective inhibitors of the small-molecule BET, namely, JQI and I-BET762, have also been proven to be effective in treating Enz-resistant CRPC by targeting the amino-terminal bromodomains of bromodomain-containing protein 4 (BRD4) (Asangani, Dommeti et al., 2014, Chan, Selth et al., 2015). In addition, later research demonstrated that the conserved zinc-finger transcription factor (ZFX) interacted with AR-V7 and co-occupied the unique AR-V7-binding sites in target gene promoters in CRPC cells. Targeting this ZFX can provide a more effective Enz-resistant CRPC treatment (Cai, Tsai et al., 2018).

Considering the difficulty in distinguishing the activity of AR-Vs from that of AR-FL, there is urgent demand for AR-V cell models that enable the identification of AR-V-specific functional and phenotypic read-outs that are not influenced by AR-FL. Kounatidou et al. (Kounatidou, Nakjang et al., 2019) established CWR22Rv1AR-EK cells in which the expression of AR-FL was abrogated and all endogenous AR-Vs were retained through CRISPR/Cas9 editing. By utilizing this model, they validated that AR-Vs regulated a DDR gene network, including an AR-FL-like 'BRCAness' signature, which was essential for cell survival after ionizing radiation treatment. Moreover, AR-Vs interacted with poly (ADP-ribose) polymerase (PARP) and depended on PARP activity for transcriptional function. Notably, suppressing PARP influenced AR-V gene expression and reduced the growth of CRPC cells. The successful establishment of this model provided a platform for accurate studies of AR-Vs in CRPC. 


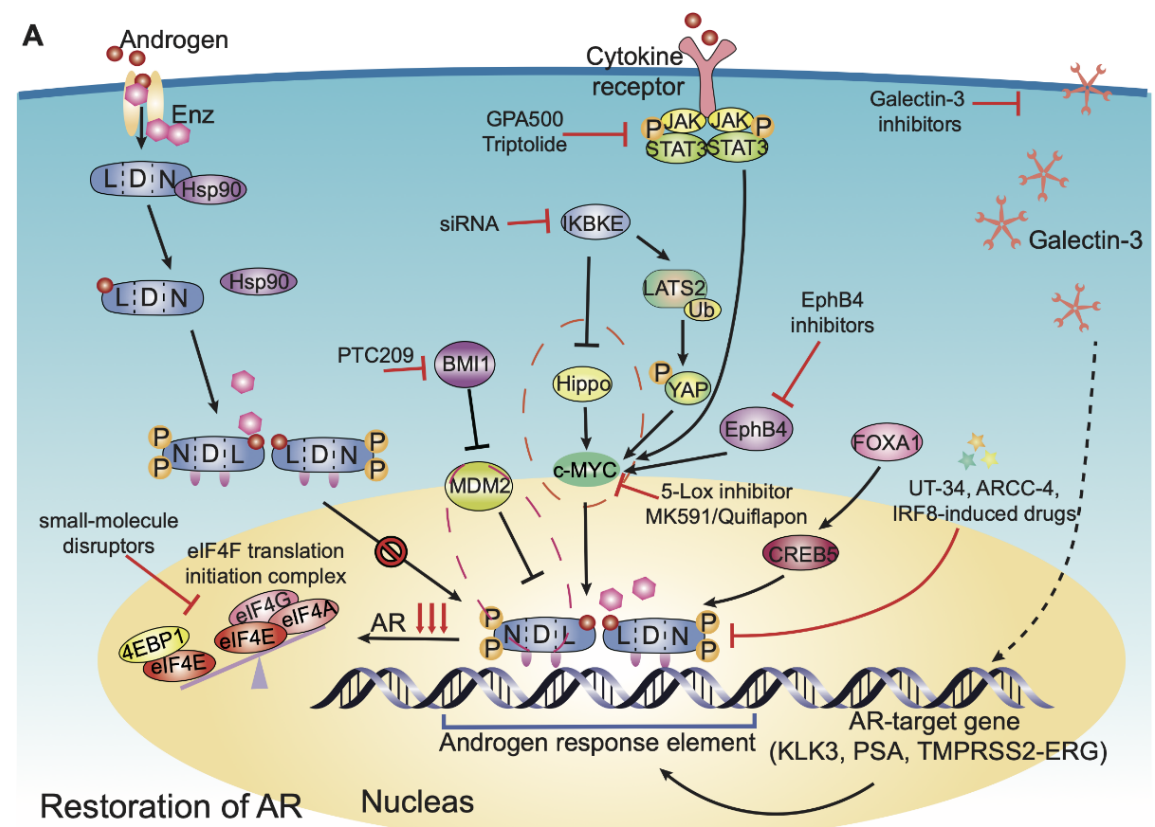

B

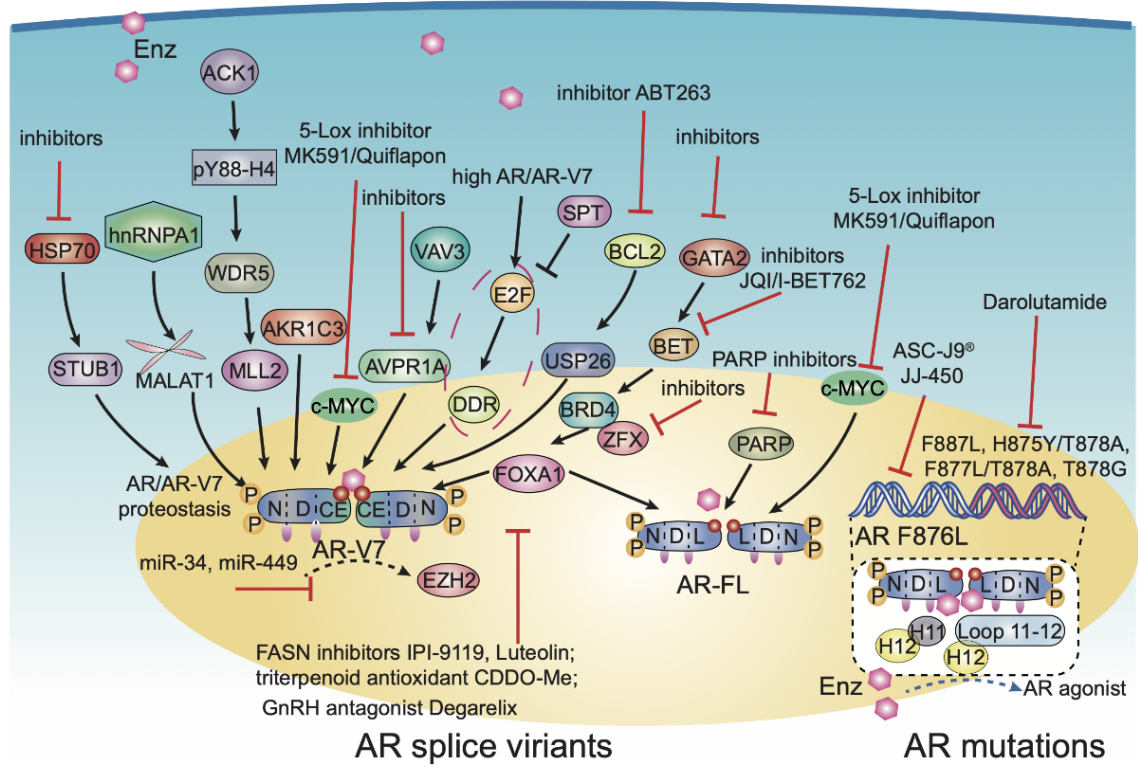

Figure 1 | Restoration of androgen receptor signaling. A | Androgen receptor reactivation. The mechanism of Enz in CRPC is shown in the left part of the cell. Various signalling pathways contribute to the activation of AR transcription and expression, involving FOXA1/CREB5/AR, IKBKE/Hippo/c-Myc/AR, and EphB4/c-Myc/AR pathways. Several inhibitors, which are associated with AR expression or targets of relevant pathways, are shown in red arrows. Especially, ARCC-4, UT-34, and IRF8 are AR degraders that directly act on AR.B | Androgen receptor splice variants and mutations. The truncated AR with a deleted LBD fails to bind with Enz. AR-V7 is the most common variant participating multiple signalling pathways, which are shown in black arrows. AR mutations contain F876L, F877L, H875Y, T878A/D891H, and T878A/S889G. Some of them can result in the alteration of Enz function that covert Enz into an agonist. Enz, 
enzalutamide; AR, androgen receptor; N, N-terminal domain; D, DNA-binding domain; L, carboxy-terminal ligand-binding domain; KLK3, kallikrein-related peptidase 3; TMPRSS2, transmembrane protease serine 2; BMI1, a polycomb group protein; PTC209, a BMI1 inhibitor; CREB5, cAMP-response element-binding protein 5; FOXA1, forkhead box protein A1; IKBKE, I-kappa-B kinase; Eph, erythropoietin-producing human hepatocellular; 4EBP1, eukaryotic initiation factor 4E-binding protein 1; eIF4F, eukaryotic translation initiation factor $4 \mathrm{~F}$, including eIF4A, eIF4E, and eIF4G; STAT3, signal transducer and activator of transcription 3; JAK, Janus kinase; GPA500, STAT3 inhibitor; Triptolide, AR/AR-Vs transactivation activity inhibitor; ARCC-4, proteolysis-targeting chimaeras (PROTACs) derivative; UT-34, the orally bioavailable AR degrader; IRF8-induced drugs, interferon regulatory factor 8; AR-V7, androgen receptor variant 7; ARFL, full-length androgen receptor; hnRNPA1, splicing factor; ACK1, non-receptor tyrosine kinases; pY88H4, Tyr88-phosphorylated histone H4; MLL2, a H3K4methyltransferase; WDR5, MLL2-interacting protein; AVPR1A, arginine vasopressin receptor 1a; VAV3, a Rho family guanosine triphosphatase guanine nucleotide exchange factor; SPT, supraphysiological testosterone; E2F, the transcription factor; DDR, DNA damage response; EZH2, zeste homologue 2; HSP, heat shock protein; STUB1, E3 ubiquitin ligase; AKR1C3, aldo-keto reductase family 1 member C3; BCL2, B cell lymphoma-2; ABT263, BCL2 protein inhibitor; USP26, ubiquitin specific protease 26; IPI-9119, fatty acid synthase inhibitor; Luteolin, AR-V7 inhibitor; GATA2, GATA binding protein 2; BET, bromodomain and extra-terminal; JQI/I-BET762, small-molecule BET inhibitor; BRD4, amino-terminal bromodomains of bromodomain-containing protein 4; ZFX, zinc-finger transcription factor; PARP, poly ADP-ribose polymerase; H12, helix 12; ASC-J9@, AR degradation enhancer; JJ-450, a $\mathrm{AR}$ antagonist.

Androgen receptor mutations

CRPC patients could be resistant to Enz because of AR mutations. The majority of mutations occur in the LBD (Snow, Lallous et al., 2019). Molecular dynamics simulations and molecular mechanics based on generalized Born surface area calculations have validated that helix 12 (H12) functions as a cover at the top of AR LBD. Under normal circumstances, the C-ring of Enz is near H12, leading to the closing of H12 and the distortion of the coactivator-binding site. When the C-ring of Enz is located near helix H11 or loop 11-12, H12 tends to serve as an auxiliary activator-binding site to promote transcription. Interestingly, in this case, Enz switches to become an AR agonist (Liu, Wang et al., 2017). For instance, the AR F876L mutation was previously found to convert the AR antagonist Enz to an AR agonist (Korpal, Korn et al., 2013). In this respect, the AR degradation enhancer ASC-J9 ${ }^{\circledR}$ directly targets AR F876L, mediating the degradation of this mutant (Wang, Lin et al., 2016). In addition, a novel AR antagonist, JJ-450, inhibited the translocation and function of the AR F876L mutant, which suggests a new potential strategy for Enz-resistant CRPC treatment (Wu, Wang et al., 2020). Other studies incorporating the detection of circulating cell-free DNA showed that the F877L, H875Y, T878A/D891H, and T878A/S889G AR mutations can lead to altered Enz functions. In particular, the double mutant F877L/T878A converted Enz into a fully functional agonist. The AR antagonist darolutamide (ODM-201), which is focused on AR mutants, significantly suppressed the transcriptional activity of the F877L, H875Y/T878A, F877L/T878A, and T878G mutants (Prekovic, Van et al., 2016 , Borgmann, Lallous et al., 2018 , Lallous, Volik et al., 2016).

\section{Glucocorticoid receptor overexpression}

The glucocorticoid receptor (GR) is one of the members of a class of nuclear steroid receptors. The expression of GR is upregulated after Enz treatment, which contributes to the re-expression of approximately $50 \%$ of the AR-responsive genes. Several canonical AR target genes have not been shown to be regulated by GR, including KLK3 and TMPRSS2. AR and GR have highly homologous DBDs and overlapping transcriptomes and cistromes. AR directly represses GR expression via a negative androgen response element (ARE) in the GR promoter. Consequently, upon exposure to the AR inhibitor Enz, the inhibition of GR expression is abrogated. The GR agonist dexamethasone is sufficient to make patients resistant to Enz, while GR antagonists can restore sensitivity (Arora, Schenkein et al., 2013).

GR plays a critical role in humans, and completely blocking GR is incompatible with life. Li et al. (Li, Alyamani et al., 2017) focused on the tumour metabolic switch associated with GR activation. They demonstrated 
that Enz-resistant prostate cancer cells sustained cortisol concentrations due to the loss of $11 \beta$-hydroxysteroid dehydrogenase-2 (11ß-HSD2) and the impaired conversion to cortisone. As a result, GR was inhibited, and glucocorticoid-dependent signalling was activated. Moreover, ubiquitin E3-ligase autocrine mobility factor receptor (AMFR) mediated the loss of $11 \beta$-HSD2, leading to Enz resistance. In a genome-wide CRISPRCas9 screening study (Palit, Vis et al., 2019), researchers found that the loss of transducin-like enhancer of split 3 (TLE3) in conjunction with AR inhibition by the GR enhancer resulted in GR upregulation, which led to Enz resistance in LNCaP cells. Hence, TLE3 was involved in the regulation of GR expression and drug resistance, providing a novel target for Enz-resistant CRPC treatment. Considering the increase in GR upon AR inhibition, Remi M et al. (Adelaiye-Ogala, Gryder et al., 2020) demonstrated that inhibiting the phosphatidylinositol 3-kinase/ protein kinase B (PI3K/AKT) pathway can block GR expression and activity after AR inhibition, overcoming GR-mediated resistance to Enz.

It was reported that BET bromodomain inhibition, pyrrole-imidazole polyamide, and selective glucocorticoid receptor modulators could inhibit GR expression and GR-regulated proliferation-associated genes for Enzresistant CRPC treatment (Shah, Wang et al., 2017 , Kurmis, Yang et al., 2017 , Kach, Long et al., 2017). Recently, Wu et al. (Wu, Xie et al., 2019) utilized a rational drug design method to analyse the chemical structures of the anti-androgen and crystal structure of GR. They developed GR/AR dual antagonists by virtual screening and biological evaluation. After performing a bioassay, they found that the dual antagonist Z19 inhibited the transcriptional activity of both AR and GR, reducing the protein and mRNA levels of the downstream proteins of GR and AR signalling (PSA for AR and KLF9 for GR). This rational drug design provides an efficient strategy for the development of novel agents for Enz-resistant CRPC

Table | Restoration of AR signalling and GR overexpression in resistant CRPC

Mechanisms

AR reactivation

AR mutations

GR overexpression
Table | Restoration of AR s

Signalling pathways/Targe AR enhancer $(650 \mathrm{~kb}$ centrom Galectin-3

BMI1

FOXA1/CREB5/AR

IKBKE/Hippo/c-Myc/AR

EphB4/c-Myc/AR

eIF4F

STAT3/AR

AR/AR-Vs

$\mathrm{AR}$

AR

lncRNA Malat1

ACK1/pY88-H4/WDR5/MLL AR-V7

AVPR1A

NK cells/microRNA-34, micro HSP70/STUB1/AR-V7

AKR1C3/AR-V7

AR-V7

c-Myc/AR-Vs, AR-FL

GATA2/AR-FL, AR-Vs

BRD4

ZFX/AR-V7

PARP/AR-V7

AR F876L mutation

F877L, H875Y, T878A/D891H

AMFR-11 $\beta$-HSD2

TLE3 


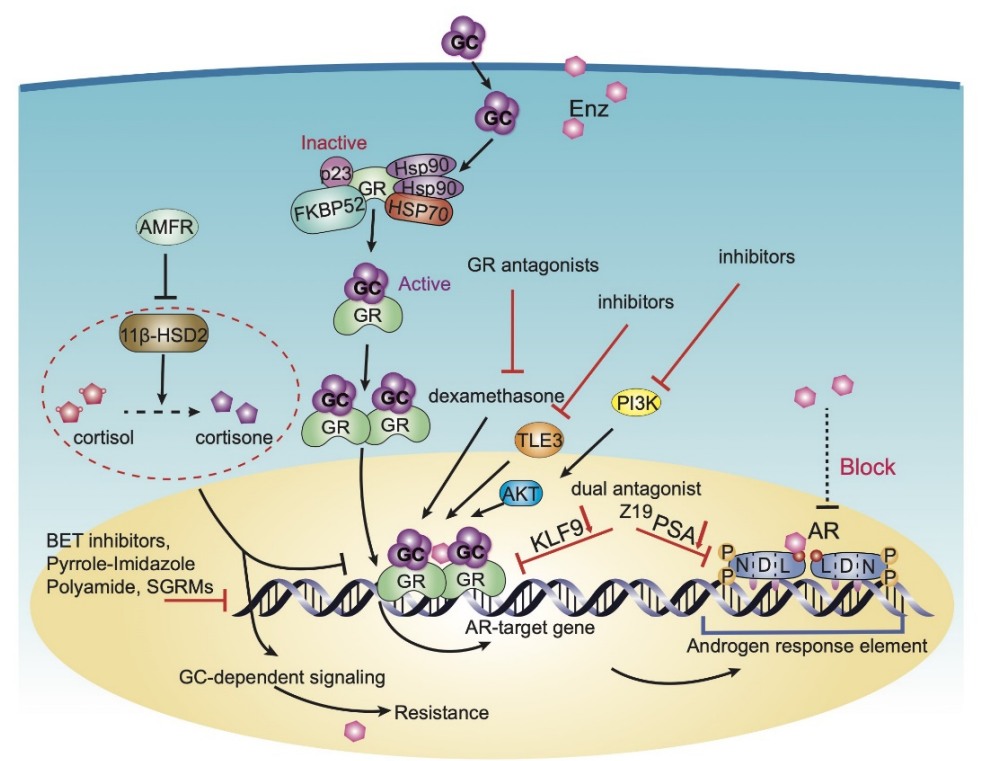

Figure 2 | Glucocorticoid receptor overexpression. The expression of GR is upregulated after inhibition of AR. AMFR mediate the loss of 11ß-HSD2 to maintain cortisol concentrations, which leads to Enz resistance. The loss of TLE3 is another reason of GR upregulation. In addition to the signal target inhibitors, GR/AR dual antagonists Z19 suppresses the transcriptional activity of both AR and GR. GR, glucocorticoid receptor; GC, glucocorticoid; p23, the small, acidic co-chaperone; FKBP52, a large peptidyl-proyl cis-trans isomerase immunophilin protein; AMFR, ubiquitin E3-ligase autocrine mobility factor receptor; 11ß-HSD2, 11ß-hydroxysteroid dehydrogenase-2; TLE3, transducin-like enhancer of split 3; PI3K/AKT, phosphatidylinositol 3-kinase/ protein kinase B; KLF9, Kruppel-like factor 9; PSA, prostate specific antigen.

\section{Activation of Wnt signalling}

Accumulating evidence suggested that Wnt signalling plays complex roles in CRPC and has therapeutic effects of AR-targeting agents. The Wnt family consists of 19 cysteines and secreted lipoglycoproteins, which regulate stem cell self-renewal and cell proliferation, migration, and differentiation during embryonic and organ development. Wnt proteins bind to multiple transmembrane frizzled (FZD) and diverse coreceptors, such as low-density lipoprotein receptor (LRP)4, LRP5, and LRP6, inactive tyrosine-protein kinase transmembrane receptor (ROR) 1 and ROR2, and tyrosine-protein kinase (RYK), to activate canonical ( $\beta$-catenin-dependent) and noncanonical ( $\beta$-catenin-independent) signalling pathways (Murillo-Garzon and Kypta, 2017).

The Wnt/ $\beta$-catenin pathway is the best-studied canonical Wnt signalling pathway. It has been reported to affect cell proliferation and differentiation and the epithelial-to-mesenchymal transition (EMT) transition in prostate cancer. The Wnt ligands form complexes with FZD receptors and coreceptors, including LRP5 or LRP6. The complex is inhibited after receptor activation, leading to a blockade of the phosphorylation of $\beta$-catenin by both casein kinase I $\alpha$ and glycogen synthase kinase-3 $\beta$ (GSK-3 $\beta$ ). $\beta$-catenin is a multifunctional protein that regulates cell adhesion and gene activation. A characteristic of canonical Wnt signalling is the 
stabilization and nuclear localization of $\beta$-catenin. Genomic and RNA-seq analyses demonstrate that Wnt $/ \beta$-catenin signalling is activated in Enz-resistant CRPC, partially due to a reduction in $\beta$-TrCP-mediated ubiquitination. Moreover, the connection between AR and $\beta$-catenin is stronger in CRPC tissues than it is in normal tissues. The upregulation of stem-like genes upon the activation of the Wnt/ $\beta$-catenin pathway contributes to Enz resistance by reactivating AR signalling (Zhang, Cheng et al., 2018). $\beta$-catenin is interacts with AR and is an AR coactivator, as indicated by $\beta$-catenin armadillo repeats directly interacting with the LBD of AR. Sex-determining region Y(Sry)-related high-mobility group (HMG) box 9 (SOX9), a member of the SOX family of HMG DNA-binding domain-containing transcription factors, regulated $\beta$-catenin/T cell factor activity and may be upregulated downstream of Wnt/ $\beta$-catenin signalling. It was induced by Wnt signalling and positively regulated multiple genes of Wnt pathways. Moreover, AR was one of the SOX9regulated proteins in PCa cells. The SOX9-AR-Wnt/ $\beta$-catenin signalling axis in PCa led to the synergistic aberrant expression of the target genes involved in cell viability, multiplication, and differentiation, resulting in the early emergence of invasive and aggressive CRPC. Therefore, targeting the SOX9-AR-Wnt/ $\beta$-catenin signalling axis may provide a potential strategy for Enz-resistant CRPC treatment (Khurana and Sikka, 2019). In addition, PRKAR2B is also a critical oncogenic gene in CRPC, which belongs to the tetrameric enzyme PKA. PRKAR2B is upregulated in CRPC cells, inducing the EMT and activating the Wnt/ $\beta$ catenin pathway. Further research indicated that miR-200b-3p, miR-200c-3p, and X-box-binding protein 1 were upstream regulators of PRKAR2B in prostate cancer. Notably, miR-200b-3p and miR-200c-3p were strongly downregulated in CRPC cells and negatively correlated with the expression of PRKAR2B. Given the multiple key factors regulating PRKAR2B, they are additional candidates for therapeutic targets for treatment (Sha, Han et al., 2018 , Xia, Han et al., 2020). The small-molecule Wnt/ $\beta$-catenin inhibitor CWP232291 inhibited $\beta$-catenin and AR, inducing endoplasmic reticulum stress and upregulating the proapoptotic transcription factor CHOP. It may also be a candidate for CRPC treatment when chemotherapy fails (Pak, Park et al., 2019).

Noncanonical Wnt signalling is divided into planar cell polarity (PCP) and Wnt-Ca ${ }^{2+}$ pathways. Upon Wnt binding to FZD receptors and tyrosine-kinase-like coreceptors, noncanonical Wnt signalling is activated, leading to the recruitment and activation of Dishevelled (DVL). The PCP pathway has two parallel branches related to small GTPases, including Rho and Rac. Rho activates Rho-associated kinase (ROCK), and Rac is linked to c-Jun N-terminal kinase (JNK). Cytoskeletal and transcriptional changes associated with these small GTPases regulate cell adhesion and migration. The $\mathrm{Wnt}_{-} \mathrm{Ca}^{2+}$ pathway stimulates $\mathrm{Ca}^{2+}$ release from the endoplasmic reticulum after being activated, thereby promoting the activation of G-proteins, protein kinase C (PKC), and $\mathrm{Ca}^{2+} /$ calmodulin-dependent kinase type II (CaMKII) (Murillo-Garzon and Kypta, 2017). RNA-seq of single prostate circulating tumour cells (CTCs) indicated the activation of the noncanonical Wnt signalling pathway induced by anti-androgens. Notably, the noncanonical Wnt ligand Wnt5A enhanced the survival of AR-positive LNCaP cells in the presence of Enz (Miyamoto, Zheng et al., 2015). Subsequent research validated that the finding that the expression of Wnt5A, RhoA, and ROCK was increased in Enzresistant PCa cells. Moreover, the combination of the ROCK inhibitor Y27632 and Enz synergistically inhibited 22RV1-derived xenograft tumour growth. Additionally, ROCK deletion combined with Enz inhibited the invasion and migration of Enz-resistant PCa cells by suppressing the EMT (Chen, Liu et al., 2020).

In addition to the activation of Wnt signalling pathways, Wnt-activating mutations may affect Enz therapy in CRPC. Since Wnt-activating mutations are present in 10-20\% of advanced prostate cancers, a recent study found that metastatic CRPC (mCRPC) patients with Wnt pathway mutations showed poor therapeutic effects after abiraterone or Enz treatment. Even after adjusting for multivariate factors and considering the alterations in other vital tumour suppressor genes, the negative prognostic impact of Wnt pathway mutations persisted. Targeting Wnt alterations may provide another idea for Enz-resistant treatment (Isaacsson Velho, Fu et al., 2020). Lombard et al. took this novel step and explored the role of Wnt-secretion-mediating proteins (Lombard, Liu et al., 2019). RNA-seq and gene set enrichment analysis were utilized to validate the expression profile changes in Enz-resistant C4-2B-MDVR cells compared to that of the parental C4-2B cells. Wntless (WLS) was needed for the secretion of all Wnts and mediated the transport of Wnts. WLS was 
found to be overexpressed in the resistant cells, regulating Wnt signalling and cellular viability. Furthermore, WLS regulated AR and AR variant expression and downstream signalling. These findings support the idea that targeting Wnt secretion may be a potential strategy for treating Enz-resistant CRPC

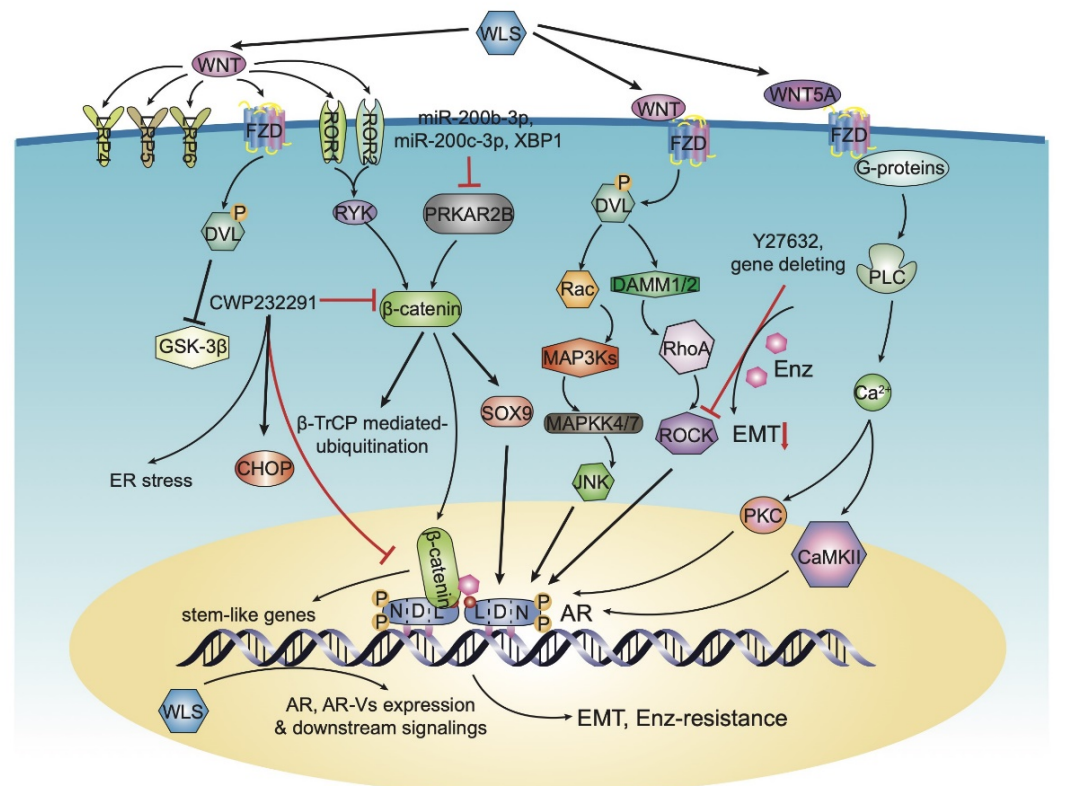

Figure 3 | Activation of Wnt signalling. Wnt proteins bind to FZD and coreceptors, including LRP4, LRP5, LRP6, ROR1, ROR2, and RYK. Canonical Wnt signalling pathway contain $\beta$-catenin and $\beta$-cateninrelated pathways while noncanonical Wnt signalling pathway include $\mathrm{Ca}^{2+}$, Rho, and Rac pathways. Notably, WLS have multiple functions in regulating Wnt and AR signalling. FZD, transmembrane frizzled; LRP, lowdensity lipoprotein receptor; ROR, tyrosine-protein kinase transmembrane receptor; RYK, tyrosine-protein kinase; GSK-3 $\beta$, glycogen synthase kinase-3 $\beta$ - SOX9, sex-determining region Y(Sry)-related high-mobility group (HMG) box 9; PRKAR2B, a oncogenic gene of tetrameric enzyme PKA; CWP232291, Wnt/ $\beta$-catenin inhibitor; CHOP, pro-apoptotic transcription factor; DVL, Dishevelled; Rho and Rac, two branches of planar cell polarity pathway; MAP3Ks, mitogen-activated protein kinase kinase kinases; MAPKK, mitogenactivated protein kinase kinase; DAAM, Dishevelled associated activator of morphogenesis; ROCK, Rhoassociated kinase; JNK, c-Jun N-terminal kinase; PKC, protein kinase C; CaMKII, Ca2+/calmodulindependent kinase type II; PLC, phospholipase C; Y27632,a ROCK inhibitor; WLS, Wntless.

\section{Glycolysis}

Normally, differentiated cells rely on mitochondrial oxidative phosphorylation to produce the energy needed for cellular processes. However, most cancer cells depend on aerobic glycolysis. This cancer-related change in metabolism is known as the "Warburg effect". Cancer cells are inclined to convert most glucose to lactate regardless of whether oxygen is present (Heiden, Cantley et al., 2009). In prostate cancer, AR regulates genes related to glucose consumption and biomass production. Moreover, androgens increase the activity of several glycolytic enzymes, such as hexokinase-2 and 6-phosphofructo-2-kinase/fructose-2,6-bisphosphatase 2. Androgens contribute to glycolysis via calcium/calmodulin-dependent protein kinase kinase beta activating AMP-activated protein kinase (AMPK) (Gonzalez-Menendez, Hevia et al., 2018). Glucose transporters (GLUTs) are upregulated in PCa cells. GLUT1 is a GLUT family member and is associated with poor prognosis (Wang, Xu et al., 2020). The GLUT1 gene promoter directly binds to AR, which promotes GLUT1 transcription. GLUT1 may act as a potential target, and the combination of a GLUT1 inhibitor and Enz may 
suppress CRPC cell proliferation and glycolysis and induce apoptosis. A previous study demonstrated that genes involved in regulating glucose metabolism were altered in LNCaP cells overexpressing NF- $x \mathrm{~B} 2 / \mathrm{p} 52$ (p52), leading to the enhancement of glucose flux for glycolysis and resistance to Enz (Cui, Nadiminty et al., 2014).

Hypoxia is the pathological feature of solid tumours and contributes to the invasion of cancers. Clinical evidence indicates that hypoxia and hypoxia-inducible factor (HIF) may play vital roles in CRPC development and treatment resistance (Bharti, Kakkad et al., 2019). Geng et al. established a molecular model in which androgen/AR independence and therapy resistance may both be due to the successful blockade of the androgen/AR axis (by ADT, Enz, or siRNA, etc.) under hypoxia (Geng, Xue et al., 2018). They found that glucose-6-phosphate isomerase (GPI) was inhibited by AR transcription under hypoxia but was recovered and increased upon AR inhibition. GPI maintained glucose metabolism and energy homeostasis under hypoxia by shifting the glucose flux from the androgen/AR-dependent pentose phosphate pathway to the hypoxia-induced glycolysis pathway, resulting in a decrease in the growth inhibition of Enz. Hence, targeting GPI may improve the therapeutic effect of Enz and is a novel method to overcome drug resistance.

\section{Induction of autophagy}

Autophagy is a highly conserved catabolic process that regulates cytoplasmic biomass, organellar abundance, and organellar distribution, removing harmful protein aggregates and intracellular toxins. Moreover, autophagy provides regenerated metabolites to promote tumour cell survival. Autophagy can be regulated in multiple ways. In nutrient-rich conditions, the PI3K/AKT pathway inhibits autophagy through the activation of the mammalian target of rapamycin (mTOR). In the absence of nutrients or growth factors or under hypoxic conditions, autophagy is activated by the AMPK pathway, resulting in the upregulation of the transcription of autophagy genes and the inhibition of mTOR/S6K/4EBP activity by the phosphorylation of TSC2. Autophagy contributes to Enz resistance through the activation of AMPK and inhibition of the mTOR pathway (Farrow, Yang et al., 2014, Smith and Macleod, 2019 , Nguyen, Yang et al., 2014).

\section{Inhibition ofapoptosis}

Apoptosis is a form of programmed cell death that plays a critical role in organism development and tissue homeostasis, resulting in the removal of damaged cells. However, cancer cells have lost their ability to undergo apoptosis, which lead to their uncontrolled proliferation. These cancer cells usually overexpress proteins related to the resistance of apoptosis cascade activation (Pistritto, Trisciuoglio et al., 2016). BCL2 proteins are frequently overexpressed in cancers and are associated with disease progression and treatment resistance. The BCL2 family contains anti-apoptotic proteins, including BCL-2, BCL-XL, MCL-1, and BCL-w, and pro-apoptotic proteins, including BAX, BAK, and BIM (Siddiqui, Ahad et al., 2015).

It has been reported that the anti-apoptotic BCL2 protein family members BCL-XL and MCL-1 are critical factors mediating Enz resistance. BCL-XL and MCL-1 bind and separate pro-apoptotic proteins BIM and BAX, resulting in the inhibition of apoptosis. Moreover, PI3K/AKT signalling is activated by Enz and can suppress BAD, a BH3-only protein that activates pro-apoptotic signalling through the inhibition of BCL-XL, mediating apoptosis evasion. However, different prostate cell lines showed differences in the expression of pro-apoptotic and anti-apoptotic BCL2 family proteins. Another study found that BCL-2 was exclusively upregulated in CRPC cells, but not BCL-XL and MCL-1, and was directly induced by Enz, which enhanced cancer stem cell (CSC)-enriched holoclones (Pilling and Hwang, 2019). The BCL-2-selective inhibitor ABT199 combined with Enz in LNCaP cells drastically inhibited Enz-resistant CRPC. Thus, targeting BCL2 protein signalling plus Enz is a promising therapeutic treatment for ENZ resistance (Li, Deng et al., 2018). The novel small molecule PAWI-2 reduced the protein levels of BCL-2, BCL-XL, and MCL-1 to normal levels in the presence of Enz. PAWI-2 can inhibit tumour growth either as a single agent or in combination with Enz to overcome Enz resistance in CRPC (Cheng, Moore et al., 2019).

The inhibitor of apoptosis protein (IAP) family also inhibits apoptosis by regulating the activity of caspases, and some IAPs are overexpressed in cancers, mediating tumour pathogenesis, progression, and resistance. The IAP family contains eight members, including NAIP (BIRC1), cIAP1 (BIRC2), cIAP2 (BIRC3), XIAP 
(BIRC4), survivin (BIRC5), Apollon/BRUCE (BIRC6), ML-IAP/LIVIN (BIRC7), and ILP-2 (BIRC8) (Krajewska, Krajewski et al., 2003). BIRC6 played a vital role in Enz resistance in a high-fidelity, Enz-resistant, patient-derived CRPC tissue xenograft model named LTL-313BR. BIRC6 is an exclusive IAP member among the eight proteins that were upregulated in both Enz-resistant systems. Furthermore, an BIRC6-targeting antisense oligonucleotide (ASO-6w2) inhibited the growth of LTL-313BR xenografts and increased the apoptosis rate (Luk, Shresth et al., 2016). Another IAP inhibitor, AEG40995, expanded the caspase-mediated apoptotic response to Enz by inducing TNF- $\alpha$ signalling. Combining Enz with IAP antagonists may overcome Enz resistance in CRPC (Pilling, Hwang et al., 2017).

\section{Lineage plasticity and phenotype switching}

Cancer cell plasticity can be defined as the ability of a cell to substantially alter its characteristics and exhibit a new phenotype that is closer to a unique developmental lineage. Cell plasticity allows tumour cells to reversibly convert to cellular properties independent of the drug-targeted pathways. The increased diversity of tumour cells is associated with therapeutic resistance and metastasis, suggesting that the retention of pluripotent progenitors and the persistence of progenitor cells can re-proliferate resistant and metastatic tumour cells with different phenotypes. PCa is driven by androgens and developsvia ligand-mediated AR signalling. Although early treatment is effective, PCa cells can adapt to androgen deprivation and restore AR signalling, eventually progressing to CRPC, which can be treated by the AR antagonist Enz. However, after long-term AR inhibition, the tumour archetype changes, which results in histological de-differentiation and cell lineage alterations in the form of the EMT and/or neuroendocrine differentiation (Davie, Beltran et al., 2018).

The EMT is a process in which the epithelial phenotype is lost and mesenchymal characteristics are acquired. The expression of EMT drivers (ZEB1, ZEB2, Snail, Twist, and FOXC2) and mesenchymal markers (Ncadherin, fibronectin, and vimentin) in PCa cells was significantly enhanced by Enz treatment, promoting the migration of $\mathrm{PCa}$ cells and inducing the transformation of prostate cancer into spindle and fibroblast-like forms (Miao, Yang et al., 2017). Transforming growth factor $\beta$ (TGF- $\beta$ ) is one of several molecular drivers contributing to the EMT process (Quintanal-Villalonga, Chan et al., 2020). RNA-seq data from CTCs have shown that TGF- $\beta$ and cyclin D1 (CCND1) signalling pathways are significantly upregulated in drug-resistant CTCs. Moreover, the key regulators of the TGF- $\beta$ pathway, namely, SMAD family member 3 (SMAD3) and CCND1, participate in the resistance to Enz (Pal, Patel et al., 2018). A new study found that the loss of the transcription factor FOXA1 led to the distinct upregulation of TGF- $\beta 3$, inducing TGF- $\beta$ signalling and the EMT. Compared with a single Enz treatment, adding the TGF- $\beta$ receptor I kinase inhibitor galunisertib (LY2157299) enhanced the efficacy of Enz in inhibiting CRPC xenograft tumour growth and metastasis (Song, Park et al., 2019 , Paller, Pu et al., 2019). The transcription factor STAT3 is also associated with the EMT and ENZ resistance. Metformin, a TGF-31/STAT3 axis inhibitor, alleviated resistance to Enz by suppressing the EMT (Liu, Tong et al., 2017). Recently, the quinazoline-derived agent DZ-50 was found to reverse EMT to MET by targeting insulin-growth factor binding protein-3 (IGFBP-3), resulting in the re-differentiation of prostate cancer. Phenotype reversal in prostate cancer cells contributes to re-sensitizing PCa cells to Enz to overcome resistance to anti-androgen therapy (Hensley, Cao et al., 2019).

Previous studies have demonstrated that the loss of the retinoblastoma tumour suppressor gene RB1 promotes lineage plasticity and the metastasis of PCa with PTEN mutations. Simultaneously losing RB1 and the tumour suppressor gene TP53 facilitated resistance to anti-androgen therapy. RB1 and TP53 suppress epigenetic reprogramming factors such as EZH2 and SOX2, which play critical roles in generating induced pluripotent stem cells (Ku, Rosario et al., 2017, Ge, Wang et al., 2020). The enhancer of EZH2 is usually overexpressed in $\mathrm{PCa}$ and associated with poor prognosis. EZH2 directly binds to the promoter of prostatespecific antigen (PSA) and suppresses its expression in Enz-resistant PCa cells. EZH2 inhibition/depletion boosts the efficacy of Enz in resistant CRPC (Xiao, Tien et al., 2018). Studies have proven that AR inhibition drives the transformation of prostate cancer into a neuroendocrine phenotype (Carceles-Cordon, Kelly et al., 2020). The understanding of neuroendocrine prostate cancer (NEPC) biology remains poorly understood. Zhang et al. (Zhang, Zheng et al., 2018) demonstrated that neuroendocrine differentiation 
(NED) and angiogenesis were regulated by ADT-activated CREB, which in turn enhanced EZH2 activity. Notably, anti-angiogenic factor thrombospondin-1(TSP1) was a direct target of EZH2 epigenetic repression. Castration activated the CREB/EZH2 axis, thereby upregulating NE markers and downregulating TSP1. The CREB/EZH2/TSP1 axis was found to be a new pathway for CRPC/NEPC treatment. The long noncoding RNA-p21 (lncRNA-p21), as a TP53 co-repressor, was upregulated upon Enz treatment to induce NED. Enz promoted the transcription of lncRNA-p21 by changing the AR binding affinity for different AREs, converting the function of EZH2 from being a histone-methyltransferase to a non-histone methyltransferase, which led to STAT3 methylation resulting in NED. The Enz/AR/lncRNA-p21/EZH2/STAT3 signalling pathway provides novel targets for developing new therapeutic methods for Enz-resistant CRPC (Luo, Wang et al., 2019). SOX2 is a reprogramming transcription factors and is upregulated after the function of TP53 and RB1 is lost, leading to drug resistance. However, previous studies did not indicate whether elevated SOX2 was sufficient to induce NE marker expression or Enz resistance (Mu, Zhang et al., 2017). By utilizing LNCaP cells engineered for the inducible elevation of SOX2, Metz et al. (MetzWilder et al., 2020) showed that increasing SOX2 did not diminish the growth inhibition of Enz or its ability to reduce the expression of PSA. Thus, the identification of the exact mechanisms of the synergistic effects of SOX2 elevation in NE plasticity and the discovery of therapeutic targets to restore cell sensitivity to Enz are urgently needed. SOX9 is a recently discovered SOX family member that is upregulated in stem-like reprogrammed prostate cancer cells. Transient SOX9 expression facilitated the resistance to Enz via NF- $x \mathrm{~B}$ dimer activation (Nouri, Massah et al., 2020). Another epigenetic remodelling regulator, the RE1-silencing transcription factor (REST), mainly binds to chromatin in the proximity of neuron-specific genes. REST silencing is a key driver of neuron-specific gene expression in prostate cancer (Flores-Morales, Bergmann et al., 2019). Serine/arginine repetitive matrix 4 (SRRM4) is an upstream regulator of REST gene functions in PCa cells, promoting the progression of NEPC. Both AR inhibition and RB1 and TP53 loss enhanced the SRRM4-induced acquisition of the neuroendocrine phenotype in prostate cancer (Li, Donmez et al., 2017). A new study (Tiwari, Manzar et al., 2020) showed that serine peptidase inhibitor Kazal type 1 (SPINK1) was transcriptionally repressed by AR and its corepressor REST. AR inhibition triggered SPINK1 upregulation, and increased SOX2 expression directly transactivated SPINK1, which facilitated the acquisition of the NE-like phenotype.

In addition, neuroendocrine lineage reprogramming is constitutively associated with MYCN. N-Myc is encoded by MYCN and overexpressed in NEPC. Epigenomic and transcriptomic reprogramming can be induced by N-Myc overexpression and its subsequent DNA binding, contributing to the lineage-plastic phenotype. Additionally, EZH2 inhibitors reversed the N-Myc-induced inhibition of epithelial lineage genes. N-Myc depended on the BET family of epigenetic readers, especially BRD4, which promote the expression of the target genes. N-Myc also overlapped with HOXB13, a lineage-specific homeodomain-containing transcription factor, located predominantly at genomic loci implicated in neural lineage specification (Dardenne, Beltran et al., 2016 , Berger, Brady et al., 2019). Nerlakanti et al. (Nerlakanti, Yao et al., 2018) found that BRD4 epigenetically promotes HOXB13 expression and binds the enhancer of HOXB13. This BRD4HOXB13 axis activated AR-independent cell cycle programmes, promoting the proliferation of CRPC. The neural transcription factor BRN2 was also a major driver of NEPC and was required for the expression of terminal NE makers and the aggressive growth of Enz-resistant CRPC tumours (Bishop, Thaper et al., 2017). Mucin 1 (MUC1) (Yasumizu, Rajabi et al., 2020) is a heterodimeric protein that suppresses AR signalling and induces BRN2 in association with the induction of MYCN and EZH2, and upregulation of the NE markers related to NEPC progression. BRN4 was found to be a novel transcription factor that drove neuroendocrine differentiation by interacting with BRN2. Enz treatment contributed to the amplified release of BRN2 and BRN4 in PCa extracellular vesicles (EVs), facilitating neuroendocrine differentiation (Bhagirath, Yang et al., 2019). The tumour suppresser protein kinase C (PKC) $\lambda / \iota$ also played a critical role in NEPC progression. The downregulation of PKC $\lambda /\llcorner$ promoted cancer cell plasticity and NEPC differentiation via the mTORC1/activating transcription factor 4 (ATF4)/PHGDH axis. This metabolic reprogramming augmented intracellular S-adenosyl methionine (SAM) levels to meet the epigenetic changes that facilitated the development of NEPC properties. Notably, the loss of PKC $\lambda / \iota$ led to the activation of mTORC1, which drove an ATF-dependent gene transcription programme that increased flux through the one-carbon pathway 
(Reina-Campos, Linares et al., 2019).

Tumour heterogeneity has been widely considered to negatively affect targeted cancer therapy, especially when genomic changes occur at the same time, which reduces the reliance on proto-oncogene drivers. In an in vivo small hairpin RNA (shRNA) screening of 730 prostate cancer genes, CHD1 loss was found to be the cause of anti-androgen resistance. ATAC-seq and RNA-seq analyses demonstrated that CHD1 loss led to overall changes in open and closed chromatin and associated transcriptomic changes, conferring resistance to Enz. Notably, four transcription factors (NR3C1, POU3F2, NR2F1, and TBX2) were found by screening to participate in Enz resistance, and they can be future therapeutic targets. To better understand the genetic and epigenetic mechanisms of neuroendocrine transformation, the CTC-derived explant (CDX) model is a distinct tool that can also be used as an effective therapeutic screening approach (Zhang, Zhou et al., 2020 , Faugeroux, Pailler et al., 2020).

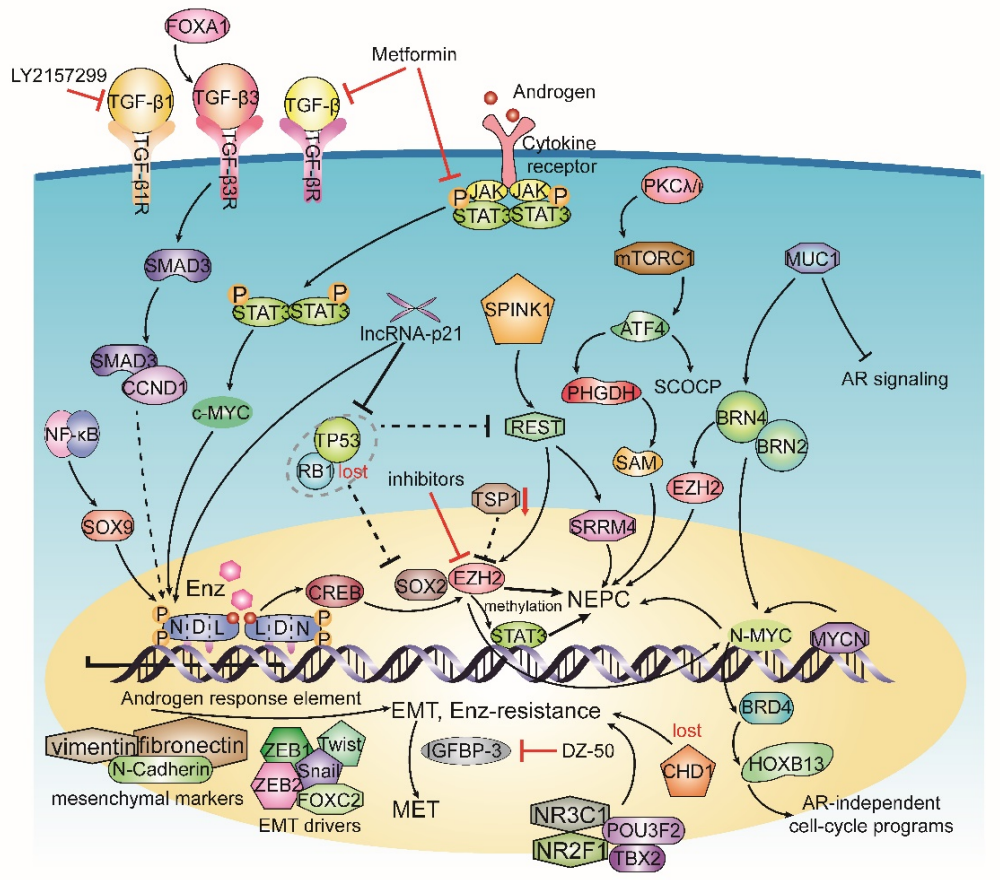

Lineage plasticity and phenotype switching

Figure $4 \mid$ Lineage plasticity and phenotype switching. After long-term AR inhibition by Enz, the phenotypes and cell lineage alter, leading to the EMT or neuroendocrine differentiation. The EMT drivers and mesenchymal markers are shown in the lower left corner of cell. TGF- $\beta$ plays a critical role in EMT process and involve diverse signalling pathways. Inhibiting TGF- $\beta$-related targets can overcome Enz resistance. The loss of RB1 and TP53 facilitates lineage plasticity and epigenetic reprogramming factors such as EZH2, SOX2, and SOX9 have effects in neuroendocrine differentiation. In particular, the loss of CHD1 contributes to anti-androgen resistance and four transcription factors (NR3C1, POU3F2, NR2F1, and TBX2) are found to participate in Enz resistance. EMT, epithelial-mesenchymal transition; TGF- $\beta$, transforming growth factor $\beta$. CCND1, cyclin D1; SMAD3, SMAD family member 3; LY2157299, TGF- $\beta$ receptor I kinase inhibitor; Metformin, a TGF- $\beta 1 /$ STAT3 axis inhibitor; IGFBP-3, insulin-growth factor binding protein3; DZ-50, IGFBP-3 inhibitor; RB1, retinoblastoma tumour suppressor gene; TP53, transformation-related protein 53; TSP1, thrombospondin-1; REST, RE1-silencing transcription factor; SRRM4, serine/arginine repetitive matrix 4; SPINK1, serine peptidase inhibitor, Kazal type 1; BRD4, bromodomain-containing 
protein 4; HOXB13, a lineage-specific homeodomain-containing transcription factor; BRN2, a neural transcription factor; MUC1, Mucin 1; (PKC) $\lambda /$, , protein kinase C; ATF4, activating transcription factor 4; PHGDH, phosphoglycerate dehydrogenase; SAM, S-adenosyl methionine; CHD1, a prostate cancer gene leads to anti-androgen resistance.

\section{Cytokine dysregulation}

Dysregulation of cytokines secreted by cancer cells, including chemokines, growth factors, pro-angiogenic factors, anti-angiogenic factors, adipokines, soluble receptors and extracellular proteases, and their associated stroma are associated with drug resistance in many diseases (Jones, Huang et al., 2016). PCa cells express autocrine interleukin-6 (IL-6), which is a frequently studied pro-inflammatory cytokine with respect to Enz resistance. IL-6 activates the Janus kinase (JAK)/STAT3 pathway, which is associated with prostate tumour growth and progression. The IL6-STAT3 axis was previously validated as a mediator of resistance to Enz, and the STAT3 inhibitor AG490 reverses ENZ resistance in CRPC (Culig and Puhr, 2018, Canesin, Krzyzanowska et al., 2020 , Liu, Zhu et al., 2014). In addition, Enz increased high mobility group box 1 (HMGB1) levels promote tumour-associated macrophage (TAM) recruitment and polarization. Activated TAMs secrete IL-6 to enhance Enz-induced NED and directly facilitate HMGB1 transcription via STAT3. Hence, blocking the feedback loop between neuroendocrine differentiation factors and TAMs by targeting IL6-STAT3 with tocilizumab and HMGB1 silencing may be a potential method for overcoming Ent resistance (Wang, Peng et al., 2018). Leukaemia inhibitory factor (LIF) and oncostatin M (OSM) are IL-6-like cytokines that are increased in the prostate epithelial cells of cancer patients. LIF activates STAT3 to promote CRPC and NED, while OSM induces morphological changes, the EMT, and migration of PCa cells via the JAK/STAT3 pathway. Similarly, IL-8 and IL-11 promote PCa cell progression through STAT3 signalling (Canesin, Krzyzanowska et al., 2020).

IL-23 is a driver of CRPC that is generated by myeloid-derived suppressor cells (MDSCs). MDSC infiltration and IL-23 were increased in blood and tumour samples from CRPC patients. IL-23 directly acted on the pSTAT3-ROR $\gamma$ axis to drive AR and its splicing variants and downstream target gene transcription. Combined, anti-IL-23 and Enz can effectively inhibit AR activity and induce tumour cell apoptosis. Thus, blocking MDSC recruitment to the tumour or inhibiting IL-23 can be potential therapeutic strategies for Enz-resistant CRPC patients (Calcinotto, Spataro et al., 2018).

The C-X-C motif chemokine ligand 12 (CXCL12) and its receptor CXCR4 are overexpressed in prostate cancer cells and tissues and are associated with tumour progression. The intracellular CXCL12 $\gamma$ isoform was found to be expressed in CTCs of m-CRPC patients and PCa cells with neuroendocrine phenotypes. The overexpression of CXCL12 $\gamma$ induced CSC and neuroendocrine phenotypesvia CXCR4-mediated protein kinase $\mathrm{C} / \mathrm{NF} \varkappa \mathrm{B}$ signalling, leading to tumour progression and drug resistance (Jung, Cackowski et al., 2018). The seven-transmembrane G protein-coupled chemokine receptor CXCR7 was the most upregulated gene in Enz-resistant CRPC and was directly regulated by AR. Elevated CXCR7 interacted with $\beta$-arrestin 2, which recruited mitogen-activated protein kinase (MAPK) proteins to phosphorylate extracellular regulated protein kinase (ERK) 1/2. MAPK/ERK inhibitors have been shown to be effective in suppressing Enzresistant CRPC. Moreover, macrophage migration inhibitory factor (MIF), a ligand for CXCR7, induced the expression of cell cycle genes by activating the AKT pathway. The MIF/CXCR7/AKT signalling pathway facilitated CRPC growth and metastasis independent of the CXCL12/CXCR4 axis. In addition, interleukin8 (IL-8) secretion and vascular endothelial growth factor (VEGF) were upregulated by CXCR7, leading to tumour angiogenesis. CXCR7 also has been reported to interact with the epidermal growth receptor (EGFR) and stimulate increased levels of phospho-EGFR (p-EGFR). The effect of the combination of CXCR7 inhibitors and Enz was manifested by significant growth inhibition of PCa cell line xenografts and PDX models of CRPC (Li, Fong et al., 2019 , Rafiei, Gui et al., 2019 , Luo, Azad et al., 2018). Similarly, other cytokines, such as fibroblast growth factor, granulocyte-macrophage colony-stimulating factor, and IL-10, were found to be elevated in the circulation of mCRPC patients resistant to Enz (Pal, Moreira et al., 2019).

\section{MicroRNAs and Enz resistance}


MicroRNAs (miRNAs) are short endogenous RNA molecules consisting of 19-25 nucleotides that regulate gene expression after transcription. It has been reported that miRNAs function as oncogenes or tumour suppressors in cancer. In particular, miRNAs have been shown to be involved in the regulation of cellular responses to drugs (Si, Shen et al., 2019). In CRPC, miR-346, miR-361-3p, and miR-197 have been proven to augment AR activity via a novel mechanism by which a $6.9-\mathrm{kb}$ AR is directly associated with the 3'UTR to stabilize the transcripts, which are increased after long-term Enz treatment (Fletcher, Sulpice et al., 2019). MicroRNA-194 (miR-194) functions as a novel post-transcriptional regulator of trans-differentiation in prostate cancer and is negatively correlated with signal-axis AR. Notably, miR-194 trans-differentiated PCa cells into a neuroendocrine-like cell by targeting FOXA1, which may contribute to Enz resistance (Fernandes, Toubia et al., 2019).

The loss of miR-101 or miR-27a during the progression of PCa contributed to the upregulation of orphan nuclear receptor COUP-TFII expression, which in turn upregulated the expression of the forkhead domain transcriptional factor FOXM1 and structural protein of kinetochore CENPF, two of the most important oncogenes in PCa. The expression of miR-101 or miR-27a was drastically decreased in Enz-resistant clones, while overexpression of miR-101 or miR-27a increased the efficacy of Enz treatment (Lin, Kao et al., 2016). The expression of miR-30c-1-3p/miR-103a-2-5p was significantly downregulated in PCa cells. MiR-30c1-3p/miR-103a-2-5p overexpression inhibited the expression of AR-V7, which was directly bound to the AR-V7 3'-UTR, suppressing CRPC cell proliferation. Importantly, the effects of miR-30c-1-3p/miR-103a2-5p overexpression was significantly reversed by AR-V7 overexpression upon Enz treatment (Chen, Yao et al., 2019). Targeting miR-30c-1-3p/miR-103a-2-5p may be a selective method of Enz-resistant therapy.

MiR-644a is a broad-spectrum cellular pathway regulator that downregulates the expression of multiple tumour microenvironment drivers, including AR coregulators, c-MYC, BCL-XL, and BCL-2. Importantly, miR-644a contributes to the downregulation of EMT markers and the elevation of E-cadherin. In addition, the Warburg effect is suppressed by miR-644a via collective inhibition of c-Myc, AKT, IGF1R, and GAPDH expression. In particular, experiments have shown that miR-644a sensitized tumours to Enz therapy. Thus, miR-644a can function as an auxiliary agent in the treatment of Enz resistance (Ebron, Shankar et al., 2019).

\section{Genepolymorphisms}

In recent years, a number of studies have focused on the association between single nucleotide polymorphisms (SNPs) in androgen-related genes and prognoses. Several SNPs also had impacts on CRPC progression and overall patient survivalc (Aragon, Cendon et al., 2019). Despite their roles in tumour development, there was a link between SNPs and Enz resistance. Y-box binding protein-1 (YB-1) is a critical factor in CRPC progression because it regulates the expression of AR-V7. Inhibiting YB-1 enhanced the effect of Enz in CRPC. The (rs12030724 polymorphism in the YB-1 gene affected the expression of YB-1 in PCa tissues (YB-1 positive rate was $14.3 \%$ in $\mathrm{TT}, 40.0 \%$ in $\mathrm{AT}$, and $52.9 \%$ in AA, $\mathrm{P}=0.04$ ) and the probability of metastatic prostate cancer progression ( $\mathrm{AT} / \mathrm{TT}$ vs $\mathrm{AA}$, hazard ratio $=0.49,95 \%$ confidence interval $=0.32$ to $0.77, \mathrm{P}=0.001$ ) (Shiota, Fujimoto et al., 2016). In another experiment, researchers investigated genetic polymorphisms in several antioxidant enzymes that were involved in regulating various stimulus-induced oxidative stress states to modulate AR and CRPC progression. Only the GSTM3 rs7483 SNP was associated with significant progression risk (AG/GG versus AA; HR; [95\% CI], $0.45[0.25-0.79], \mathrm{P}=0.0047)$. The levels of intracellular reactive oxygen species and GSTM3 expression were upregulated in both castration-resistant and Enz-resistant cells (Shiota, Fujimoto et al., 2017 , Fujimoto, Shiota et al., 2017).

According to an ARMS-PCR analysis of the androgen receptor-coding gene in 50 patients with drug-resistant $\mathrm{PCa}$ and 50 patients with non-drug-resistant $\mathrm{PCa}$ in the human population of the Isfahan province, Enz resistance was significantly correlated with the rs137852595 SNP (P-value $=0.005)$ and rs137852574 SNP $(\mathrm{P}$-value $=0.039)$. In patients with these SNPs, the binding of the drug to the active site of AR was inaccurate, leading to a reduction in the binding energy of the drugs with the receptors (Alizadeh, Sazegar et al., 2018 , Kaviani, Sazgar et al., 2018).

\section{Other mechanisms responsible for Enz-resistant CRPC}


RNA sequencing has shown that the NOTCH signalling pathways were associated with Enz resistance, which was independent of AR pathways. Notably, NOTCH1 signalling was activated in Enz-resistant cells, and inhibition of this pathway restored Enz function in vitro and in vivo (Farah, Li et al., 2019). In another short hairpin RNA (shRNA) screen of the bone-homing C4-2B CRPC cell line, several genes were found to be involved in the response to Enz. Three candidate genes, acetyl-CoA acetyltransferase 1 (ACAT1), mixed-lineage kinase 3 (MLK3), and PSMD12, which encodes the proteasome $26 \mathrm{~S}$ subunit, were validated as supporters of Enz resistance in vitro. Further studies need to be conducted to verify the drivers of these genes and the level of in vivo resistance the confer (Kohrt, Awadallah et al., 2020). Moreover, an online database search and bioinformatics analysis showed that bone morphogenetic protein (BMP)-6/SMAD signalling was activated in Enz-resistant LNCaP cells. Further study indicated the role of phospholipase C (PLC) $\varepsilon$ in regulating this pathway. Targeting the PLC 8 /BMP-6/SMAD pathway may be an alternative tactic to increase cell sensitivity to Enz (Yuan, Gao et al., 2019).

A key enzyme in cholesterol synthesis, 3-hydroxy-3-methyl-glutaryl-CoA reductase (HMGCR), has been found to be upregulated in Enz-resistant PCa cells. Aberrant HMGCR expression mediated Enz resistance of CRPC cells. Simvastatin alone or in combination with Enz in vitro and in vivo can inhibit the proliferation of Enz-resistant PCa cells via the suppression of the mTOR pathway, resulting in AR or AR-V degradation. Furthermore, cancer-associated fibroblasts (CAFs) induced a remarkable upregulation of cholesterol and steroid biosynthesis pathways in PCa cells. In addition, AKR1C3 and 3-hydroxy-methylglutaryl CoA synthase 2 (HMGCS2) were found to be significantly upregulated in PCa cells upon coculture with CAFs. The combination of simvastatin and AKR1C3 inhibitors showed the strongest growth inhibition, suggesting that the dual inhibition of cholesterol and steroid biosynthesis may be a promising method to overcome AR targeted therapy resistance (Kong, Cheng et al., 2018, Neuwirt, Bouchal et al., 2020).

\section{Discussion}

Patients with CRPC usually have a poor prognosis, and several drugs have been demonstrated to prolong overall survival, including enzalutamide and abiraterone. However, CRPC patients will eventually become resistant to enzalutamide treatment via AR-dependent or AR-independent mechanisms (Buttigliero, Tucci et al., 2015). In this review, emerging resistance mechanisms based on previous studies were elaborated, including AR signalling pathways, GR-related pathways, WNT, and metabolic mechanisms. Notably, the roles of microRNAs and gene polymorphisms in Enz resistance were emphasized. Some corresponding therapeutic measures were also introduced to provide references for the selection of therapeutic regimens.

The emergence of new technologies, such as high-throughput next-generation sequencing and liquid biopsies, will facilitate the discovery of more drug resistance genes and determine enzalutamide-resistant mechanisms more quickly (Xu, Chen et al., 2019). In addition, the establishment of CRPC patient-derived xenografts (PDXs) from patients resistant to conventional ADT, second-generation AR inhibitors, and chemotherapy are beneficial for finding more effective and specific therapeutic regimens. Notably, all these models, including an AR-null tumour with NE features, were sensitive to dual inhibition of ribosome production and function (Lawrence, Obinata et al., 2018). Notably, prostate cancer is a heterogeneous disease in which different levels of AR expression influence the sensitivity of cells to enzalutamide. Xenograft models indicated that low/no expression $\mathrm{AR}^{-/ 10} \mathrm{CRPC}$ cells were resistant to enzalutamide, while AR-expressing $\left(\mathrm{AR}^{+}\right) \mathrm{CRPC}$ cells were Enz sensitive. Patients with $\mathrm{CRPC}$ had both $\mathrm{AR}^{+/ \mathrm{hi}}$ and $\mathrm{AR}^{-/ l o} \mathrm{PCa}$ cells, which should be considered during Enz-resistant treatment (Li, Deng et al., 2018). Given the failure to direct AR-targeted therapies, selective targeted poly(ADP-ribose) polymerase-2 can provide an alternative therapeutic strategy for AR inhibition by disrupting FOXA1 binding rather than directly targeting AR(Gui, Gui et al., 2019).

In conclusion, these emerging mechanisms offer diverse angles for approaching Enz resistance and provide multiple therapeutic regimens for resistant CRPC treatments. Notably, tumour heterogeneity ,individual gene polymorphisms, and the participation of the formation of distinct drug resistance characteristics should be further explored in future studies. Additionally, combination therapies may become potent strategies for Enz-resistant CRPC treatment. 


\section{Acknowledgment}

This work was supported by the National Natural Science Foundation of China (No. 81672545, 81972392 and 81972391).

\section{Conflicts of Interest}

The authors declare no competing financial interest.

\section{References}

Rawla P. (2019). Epidemiology of prostate cancer. World J Oncol, 10(2): 63-89.

Trewartha D, Carter K. (2013). Advances in prostate cancer treatment. Nat Rev Drug Discov, 12(11): 823-824.

Zong Y, Goldstein AS. 2013. Adaptation or selection-mechanisms of castration-resistant prostate cancer. Nat Rev Urol, 10(2): 90-98.

R S. (2002). A history of prostate cancer treatment. Nat Rev Cancer, 2: 389-396.

Davies A, Conteduca V, Zoubeidi A, Beltran H. (2019). Biological evolution of castration-resistant prostate cancer. Eur Urol Focus, 5(2): 147-154.

Saad F, Chi KN, Finelli A, Hotte SJ, Izawa J, Kapoor A, . . Fleshner NE. (2015). The 2015 CUA-CUOG guidelines for the management of castration-resistant prostate cancer (CRPC). Can Urol Assoc J, 9(3-4): 90-96.

Cai C, Balk SP. (2011). Intratumoral androgen biosynthesis in prostate cancer pathogenesis and response to therapy. Endocr Relat Cancer, 18(5): R175-182.

Tran C, Ouk S, Clegg NJ, Chen Y, Watson PA, Arora V, . . . Sawyers CL. (2009). Development of a second-generation antiandrogen for treatment of advanced prostate cancer. Science, 324: 787-790.

Nelson WG, Yegnasubramanian S. (2013). Resistance emerges to second-generation antiandrogens in prostate cancer. Cancer Discov, 3(9): 971-974.

Erdogan B. (2018). Enzalutamide and cancer. EJMO, 2(3): 121-129.

Jiang W, Chen J, Gong C, Wang Y. (2020). Intravenous delivery of enzalutamide based on high drug loading multifunctional graphene oxide nanoparticles for castration-resistant prostate cancer therapy. J Nanobiotechnology, 18(1): 50.

Niu Y, Guo C, Wen S, Tian J, Luo J, Wang K, . . Chang C. (2018). ADT with antiandrogens in prostate cancer induces adverse effect of increasing resistance, neuroendocrine differentiation and tumor metastasis. Cancer Lett, 439: 47-55.

Wong YN, Ferraldeschi R, Attard G, De Bono J. (2014). Evolution of androgen receptor targeted therapy for advanced prostate cancer. Nat Rev Clin Oncol, 11(6): 365-376.

Yuan X, Cai C, Chen S, Chen S, Yu Z, Balk SP. (2014). Androgen receptor functions in castration-resistant prostate cancer and mechanisms of resistance to new agents targeting the androgen axis. Oncogene, 33(22): $2815-2825$.

Takeda DY, Spisák S, Seo JH, Bell C, O'connor E, Korthauer K, . . Freedman ML. (2018). A somatically acquired enhancer of the androgen receptor is a noncoding driver in advanced prostate cancer. Cell, 174(2): $422-432$.

Dondoo TO, Fukumori T, Daizumoto K, Fukawa T, Kohzuki M, Kowada M, . . Kanayama HO. (2017). Galectin-3 is implicated in tumor progression and resistance to anti-androgen drug through regulation of androgen receptor signaling in prostate cancer. Anticancer Res, 37(1): 125-134. 
Zhu S, Zhao D, Yan L, Jiang W, Kim JS, Gu B, . . Cao Q. (2018). BMI1 regulates androgen receptor in prostate cancer independently of the polycomb repressive complex 1. Nat Commun, 9(1): 500.

Hwang JH, Seo JH, Beshiri ML, Wankowicz S, Liu D, Cheung A, . . Hahn WC. (2019). CREB5 promotes resistance to androgen-receptor antagonists and androgen deprivation in prostate cancer. Cell Rep, 29(8): 2355-2370.

Bai S, Cao S, Jin L, Kobelski M, Schouest B, Wang X, . . . Dong Y. (2019). A positive role of c-Myc in regulating androgen receptor and its splice variants in prostate cancer. Oncogene, 38(25): 4977-4989.

Bainbridge A, Walker S, Smith J, Patterson K, Dutt A, Ng Ym, . . Coffey K. (2020). IKBKE activity enhances AR levels in advanced prostate cancer via modulation of the Hippo pathway. Nucleic Acids Res, 48(10):5366-5382.

Li C, Lanman NA, Kong Y, He D, Mao F, Farah E, . . Liu X. (2020). Inhibition of the erythropoietinproducing receptor EPHB4 antagonizes androgen receptor overexpression and reduces enzalutamide resistance. J Biol Chem, 295(16): 5470-5483.

Liu Y, Horn JL, Banda K, Goodman AZ. (2019). The androgen receptor regulates a druggable translational regulon in advanced prostate cancer. Sci Transl Med, 11: 1-12.

Thaper D, Vahid S, Kaur R, Kumar S, Nouruzi S, Bishop JL, . . Zoubeidi A. (2018). Galiellalactone inhibits the STAT3/AR signaling axis and suppresses Enzalutamide-resistant Prostate Cancer. Sci Rep, 8(1):17307.

Han Y, Huang W, Liu J, Liu D, Cui Y, Huang R, . . . Lei M. (2017). Triptolide inhibits the ar signaling pathway to suppress the proliferation of enzalutamide resistant prostate cancer cells. Theranostics, 7(7): 1914-1927.

Pollock JA, Wardell SE, Parent AA, Stagg DB, Ellison SJ, Alley HM, . . Norris JD. (2016). Inhibiting androgen receptor nuclear entry in castration-resistant prostate cancer. Nat Chem Biol, 12(10): 795-801.

Toure M, Crews CM. (2016). Small-Molecule PROTACS: New approaches to protein degradation. Angew Chem Int Ed Engl, 55(6): 1966-1973.

Salami J, Alabi S, Willard RR, Vitale NJ, Wang J, Dong H, . . . Crews CM. (2018). Androgen receptor degradation by the proteolysis-targeting chimera ARCC-4 outperforms enzalutamide in cellular models of prostate cancer drug resistance. Commun Biol, 1: 100.

Ponnusamy S, He Y, Hwang DJ, Thiyagarajan T, Houtman R, Bocharova V, . . Narayanan R. (2019). Orally bioavailable androgen receptor degrader, potential next-generation therapeutic for enzalutamide-resistant prostate cancer. Clin Cancer Res, 25(22): 6764-6780.

Wu H, You L, Li Y, Zhao Z, Shi G, Chen Z, . . Yang Y. (2020). Loss of a negative feedback loop between IRF8 and AR promotes prostate cancer growth and enzalutamide resistance. Cancer Res, 80(13):2927-2939.

J G, Bubley, Balk SP. 2017. Association between androgen receptor splice variants and prostate cancer resistance to abiraterone and enzalutamide. J Clin Oncol, 35(19): 2103-2105.

Antonarakis ES, Lu C, Wang H, Luber B, Nakazawa M, Roeser JC, . . Luo J. (2014). AR-V7 and resistance to enzalutamide and abiraterone in prostate cancer. N Engl J Med, 371(11): 1028-1038.

Stone L. (2017). Prostate cancer: Escaping enzalutamide: Malat1 contributes to resistance. Nat Rev Urol, 14(8): 450 .

Mahajan K, Malla P, Lawrence HR, Chen Z, Kumar-Sinha C, Malik R, . . Mahajan NP. (2017). ACK1/TNK2 regulates histone H4 Tyr88-phosphorylation and AR gene expression in castration-resistant prostate cancer. Cancer Cell, 31(6): 790-803

Zhao N, Peacock SO, Lo CH, Heidman LM, Rice MA. (2019). Arginine vasopressin receptor 1a is a therapeutic target for castration-resistant prostate cancer. Sci Transl Med, 11(498):eaaw4636 
Lam HM, Nguyen HM, Labrecque MP, Brown LG, Coleman IM, Gulati R, . . Corey E. (2020). Durable response of enzalutamide-resistant prostate cancer to supraphysiological testosterone is associated with a multifaceted growth suppression and impaired DNA damage response transcriptomic program in patientderived xenografts. Eur Urol, 77(2): 144-155.

Lin SJ, Chou FJ, Li L, Lin CY, Yeh S, Chang C. (2017). Natural killer cells suppress enzalutamide resistance and cell invasion in the castration resistant prostate cancer via targeting the androgen receptor splicing variant 7 (ARv7). Cancer Lett, 398: 62-69.

Liu C, Lou W, Yang JC, Liu L, Armstrong CM, Lombard AP, . . . Gao AC (2018). Proteostasis by STUB1/HSP70 complex controls sensitivity to androgen receptor targeted therapy in advanced prostate cancer. Nat Commun, 9(1): 4700.

Liu C, Yang JC, Armstrong CM, Lou W, Liu L, Qiu X, . . Gao AC (2019). AKR1C3 promotes AR-V7 protein stabilization and confers resistance to AR-targeted therapies in advanced prostate cancer. Mol Cancer Ther, 18(10): 1875-1886.

Xu H, Sun Y, Huang CP, You B, Ye D, Chang C. (2020). Preclinical study using ABT263 to increase enzalutamide sensitivity to suppress prostate cancer progression via targeting BCL2/ROS/USP26 axis through altering ARv7 protein degradation. Cancers (Basel), 12(4):831.

Zadra G, Ribeiro CF, Chetta P, Ho Y, Cacciatore S, Gao X, . . Loda M. (2019). Inhibition of de novo lipogenesis targets androgen receptor signaling in castration-resistant prostate cancer. Proc Natl Acad Sci U S A, 116(2): 631-640.

Naiki-Ito A, Naiki T, Kato H, Iida K, Etani T, Nagayasu Y, . . Takahashi S. (2019). Recruitment of miR-8080 by luteolin inhibits androgen receptor splice variant 7 expression in castration-resistant prostate cancer. Retrieved from https://doi.org/10.1093/carcin/bgz193.

Khurana N, Chandra PK, Kim H, Abdel-Mageed AB, Mondal D, Sikka SC. (2020). Bardoxolone-methyl (CDDO-Me) suppresses androgen receptor and its splice-variant AR-V7 and enhances efficacy of enzalutamide in prostate cancer cells. Antioxidants (Basel), 9(1):68.

Cucchiara V, Yang JC, Liu C, Adomat HH, Tomlinson Guns ES, Gleave ME, . . Evans CP. (2019). GnRH antagonists have direct inhibitory effects on castration-resistant prostate cancer via intracrine androgen and AR-V7 expression. Mol Cancer Ther, 18(10): 1811-1821.

Monga J, Subramani D, Bharathan A, Ghosh J. (2020). Pharmacological and genetic targeting of 5lipoxygenase interrupts c-Myc oncogenic signaling and kills enzalutamide-resistant prostate cancer cells via apoptosis. Sci Rep, 10(1): 6649.

Chaytor L, Simcock M, Nakjang S, Heath R, Walker L, Robson C, . . Gaughan L. (2019). The pioneering role of GATA2 in androgen receptor variant regulation is controlled by bromodomain and extraterminal proteins in castrate-resistant prostate cancer. Mol Cancer Res, 17(6): 1264-1278.

He B, Lanz RB, Fiskus W, Geng C, Yi P, Hartig SM, . . . Mitsiades N. (2014). GATA2 facilitates steroid receptor coactivator recruitment to the androgen receptor complex. Proc Natl Acad Sci U S A, 111(51): 18261-18266.

Asangani IA, Dommeti VL, Wang X, Malik R, Cieslik M, Yang R, . . Chinnaiyan AM. (2014). Therapeutic targeting of BET bromodomain proteins in castration-resistant prostate cancer. Nature, 510(7504): 278-282.

Chan SC, Selth LA, Li Y, Nyquist MD, Miao L, Bradner JE, . . Dehm SM. (2015). Targeting chromatin binding regulation of constitutively active AR variants to overcome prostate cancer resistance to endocrinebased therapies. Nucleic Acids Res, 43(12): 5880-5897.

Cai L, Tsai YH, Wang P, Wang J, Li D, Fan H, ... Wang GG. (2018). ZFX Mediates non-canonical oncogenic functions of the androgen receptor splice variant 7 in castrate-resistant prostate cancer. Mol Cell, 
72(2): $341-354$

Kounatidou E, Nakjang S, Mccracken SR C, Dehm SM, Robson CN, Jones D, Gaughan L. (2019). A novel CRISPR-engineered prostate cancer cell line defines the AR-V transcriptome and identifies PARP inhibitor sensitivities. Nucleic Acids Res, 47(11): 5634-5647.

Snow O, Lallous N, Singh K, Lack N, Rennie P, Cherkasov A. (2019). Androgen receptor plasticity and its implications for prostate cancer therapy. Cancer Treat Rev, 81: 101871.

Liu H, Wang L, Tian J, Li J, Liu H. (2017). Molecular dynamics studies on the enzalutamide resistance mechanisms induced by androgen receptor mutations. J Cell Biochem, 118(9): 2792-2801.

Korpal M, Korn JM, Gao X, Rakiec DP, Ruddy DA, Doshi S, . . Zhu P. (2013). An F876L mutation in androgen receptor confers genetic and phenotypic resistance to MDV3100 (enzalutamide). Cancer Discov, 3(9): 1030-1043.

Wang R, Lin W, Lin C, Li L, Sun Y, Chang C. (2016). ASC-J9® suppresses castration resistant prostate cancer progression via degrading the enzalutamide-induced androgen receptor mutant AR-F876L. Cancer Lett, 379(1): 154-160.

Wu Z, Wang K, Yang Z, Pascal LE, Nelson Jb, Takubo K, . . Wang Z. (2020). A novel androgen receptor antagonist JJ-450 inhibits enzalutamide-resistant mutant AR(F876L) nuclear import and function. Prostate, 80(4): 319-328.

Prekovic S, Van Royen ME, Voet AR, Geverts B, Houtman R, Melchers D, . . Helsen C. (2016). The effect of F877L and T878A mutations on androgen receptor response to enzalutamide. Mol Cancer Ther, 15(7): 1702-1712.

Borgmann H, Lallous N, Ozistanbullu D, Beraldi E, Paul N, Dalal K, . . . Gleave ME. (2018). Moving towards precision urologic oncology: targeting enzalutamide-resistant prostate cancer and mutated forms of the androgen receptor using the novel inhibitor darolutamide (ODM-201). Eur Urol, 73(1): 4-8.

Lallous N, Volik SV, Awrey S, Leblanc E, Tse R, Murillo J, . . Cherkasov A. (2016). Functional analysis of androgen receptor mutations that confer anti-androgen resistance identified in circulating cell-free DNA from prostate cancer patients. Genome Biol, 17: 10.

Arora VK, Schenkein E, Murali R, Subudhi SK, Wongvipat J, Balbas MD, . . Sawyers CL. (2013). Glucocorticoid receptor confers resistance to antiandrogens by bypassing androgen receptor blockade. Cell, 155(6): 1309-1322.

Li J, Alyamani M, Zhang A, Chang KH, Berk M, Li Z, . . Sharifi N. (2017). Aberrant corticosteroid metabolism in tumor cells enables GR takeover in enzalutamide resistant prostate cancer. Elife, 6:e20183.

Palit SA, Vis D, Stelloo S, Lieftink C, Prekovic S, Bekers E, . . . Van Der Heijden MS. (2019). TLE3 loss confers AR inhibitor resistance by facilitating GR-mediated human prostate cancer cell growth. Elife, 8:e47430.

Adelaiye-Ogala RM, Gryder B, Nguyen YTM, Alilin An, Grayson A, Jansson KH, . . Vanderweele DJ. (2020). Targeting the PI3K/AKT pathway overcomes enzalutamide resistance by inhibiting induction of the glucocorticoid receptor. Mol Cancer Ther, 19(7):1436-1447.

Shah N, Wang P, Wongvipat J, Karthaus WR, Abida W, Armenia J, . . Sawyers CL. (2017). Regulation of the glucocorticoid receptor via a BET-dependent enhancer drives antiandrogen resistance in prostate cancer. Elife, 6: 1-19.

Kurmis AA, Yang F, Welch TR, Nickols NG, Dervan PB. (2017). A Pyrrole-Imidazole Polyamide is active against enzalutamide-resistant prostate cancer. Cancer Res, 77(9): 2207-2212. 
Kach J, Long TM, Selman P, Tonsing-Carter EY, Bacalao MA, Lastra RR, . . Szmulewitz RZ. (2017). Selective glucocorticoid receptor modulators (SGRMs) delay castrate-resistant prostate cancer growth. Mol Cancer Ther, 16(8): 1680-1692.

Wu M, Xie Y, Cui X, Huang C, Zhang R, He Y, . . Zhou J. (2019). Rational drug design for androgen receptor and glucocorticoids receptor dual antagonist. Eur J Med Chem, 166: 232-242.

Tummala R, Lou W, Gao AC, Nadiminty N. (2017). Quercetin targets hnRNPA1 to overcome enzalutamide resistance in prostate cancer cells. Mol Cancer Ther, 16(12):2770-2779.

Zhao M, Wang S, Li Q, Ji Q, Guo P, Liu X. (2018). MALAT1: A long non-coding RNA highly associated with human cancers. Oncol Lett, 16(1):19-26.

Joseph JD, Lu N, Qian J, Sensintaffar J, Shao G, Brigham D, . . Hager JH. (2013). A clinically relevant androgen receptor mutation confers resistance to second-generation antiandrogens enzalutamide and ARN509. Cancer Discov, 3(9):1020-1029

Murillo-Garzon V, Kypta R. (2017). WNT signalling in prostate cancer. Nat Rev Urol, 14(11): 683-696.

Zhang Z, Cheng L, Li J, Farah E, Atallah NM, Pascuzzi PE, . . Liu X. (2018). Inhibition of the Wnt/betacatenin pathway overcomes resistance to enzalutamide in castration-resistant prostate cancer. Cancer Res, 78(12): 3147-3162.

Khurana N, Sikka SC. (2019). Interplay Between SOX9, Wnt/beta-catenin and androgen receptor signaling in castration-resistant prostate cancer. Int J Mol Sci, 20(9):2066.

Sha J, Han Q, Chi C, Zhu Y, Pan J, Dong B, . . Xue W. (2018). PRKAR2B promotes prostate cancer metastasis by activating Wnt/beta-catenin and inducing epithelial-mesenchymal transition. J Cell Biochem, 119(9): 7319-7327.

Xia L, Han Q, Chi C, Zhu Y, Pan J, Dong B, . . Sha J. (2020). Transcriptional regulation of PRKAR2B by miR-200b-3p/200c-3p and XBP1 in human prostate cancer. Biomed Pharmacother, 124: 109863.

Pak S, Park S, Kim Y, Park JH, Park CH, Lee KJ, . . Ahn H. (2019). The small molecule Wnt/ß-catenin inhibitor CWP232291 blocks the growth of castration-resistant prostate cancer by activating the endoplasmic reticulum stress pathway. J Exp Clin Cancer Res, 38(1): 342.

Miyamoto DT, Zheng Y, Wittner BS, Lee RJ. (2015). RNA-Seq of single prostate CTCs implicates noncanonical Wnt signaling in antiandrogen resistance. Science, 349(6254): 1351-1356.

Chen X, Liu J, Cheng L, Li C, Zhang Z, Bai Y, . . . Liu X. (2020). Inhibition of noncanonical Wnt pathway overcomes enzalutamide resistance in castration-resistant prostate cancer. Prostate, 80(3): 256-266.

Isaacsson Velho P, Fu W, Wang H, Mirkheshti N, Qazi F, Lima FAS, . . . Antonarakis ES. (2020). Wntpathway activating mutations are associated with resistance to first-line abiraterone and enzalutamide in castration-resistant prostate cancer. Eur Urol, 77(1): 14-21.

Lombard AP, Liu C, Armstrong CM, D'Abronzo LS. (2019). Wntless promotes cellular viability and resistance to enzalutamide in castration resistant prostate cancer cells. Am J Clin Exp Urol, 7(4): 203-214.

Heiden MGV, Cantley LC, Thompson CB. (2009). Understanding the warburg effect:The Metabolic requirements of cell proliferation. Science, 324: 1029-1033.

Gonzalez-Menendez P, Hevia D, Mayo JC, Sainz RM. (2018). The dark side of glucose transporters in prostate cancer: Are they a new feature to characterize carcinomas? Int J Cancer, 142(12): 2414-2424.

Wang J, Xu W, Wang B, Lin G, Wei Y, Abudurexiti M, . . . Ye D. (2020). GLUT1 is an AR target contributing to tumor growth and glycolysis in castration-resistant and enzalutamide-resistant prostate cancers. Cancer Lett, 485:45-55. 
Cui Y, Nadiminty N, Liu C, Lou W, Schwartz CT, Gao AC. (2014). Upregulation of glucose metabolism by NF-kappaB2/p52 mediates enzalutamide resistance in castration-resistant prostate cancer cells. Endocr Relat Cancer, 21(3): 435-442.

Bharti SK, Kakkad S, Danhier P, Wildes F, Penet MF, Krishnamachary B, Bhujwalla ZM. (2019). Hypoxia patterns in primary and metastatic prostate cancer environments. Neoplasia, 21(2): 239-246.

Geng H, Xue C, Mendonca J, Sun XX, Liu Q, Reardon PN, . . . Qian DZ. (2018). Interplay between hypoxia and androgen controls a metabolic switch conferring resistance to androgen/AR-targeted therapy. Nat Commun, 9(1): 4972.

Farrow JM, Yang JC, Evans CP. (2014). Autophagy as a modulator and target in prostate cancer. Nat Rev Urol, 11(9): 508-516.

Smith AG, Macleod KF. (2019). Autophagy, cancer stem cells and drug resistance. J Pathol, 247(5): 708-718.

Nguyen HG, Yang JC, Kung HJ, Shi XB, Tilki D, Lara PN, JR., . . . Evans CP. (2014). Targeting autophagy overcomes Enzalutamide resistance in castration-resistant prostate cancer cells and improves therapeutic response in a xenograft model. Oncogene, 33(36): 4521-4530.

Pistritto G, Trisciuoglio D, Ceci C, Garufi A. (2016). Apoptosis as anticancer mechanism: function and dysfunction of its modulators and targeted therapeutic strategies. Aging, 8(4): 603-619.

Siddiqui WA, Ahad A, Ahsan H. (2015). The mystery of BCL2 family: Bcl-2 proteins and apoptosis: an update. Arch Toxicol, 89(3): 289-317.

Pilling AB, Hwang C. (2019). Targeting prosurvival BCL2 signaling through Akt blockade sensitizes castration-resistant prostate cancer cells to enzalutamide. Prostate, 79(11): 1347-1359.

Li Q, Deng Q, Chao HP, Liu X, Lu Y, Lin K, . . . Tang DG. (2018). Linking prostate cancer cell AR heterogeneity to distinct castration and enzalutamide responses. Nat Commun, 9(1): 3600.

Cheng J, Moore S, Gomez-Galeno J, Lee DH, Okolotowicz KJ, Cashman JR. (2019). A novel small molecule inhibits tumor growth and synergizes effects of enzalutamide on prostate cancer. J Pharmacol Exp Ther, 371(3): 703-712.

Krajewska M, Krajewski S, Banares S, Huang X. (2003). Elevated expression of inhibitor of apoptosis proteins in prostate cancer. Clin Cancer Res, 9(13): 4914-4925.

Luk ISU, Shresth R, Xue H, Wang Y. 2016. BIRC6-targeting as potential therapy for advanced, enzalutamide-resistant prostate cancer. Clin Cancer Res, 23(6): 1542-1551.

Pilling AB, Hwang O, Boudreault A, Laurent A, Hwang C. (2017). IAP antagonists enhance apoptotic response to enzalutamide in castration-resistant prostate cancer cells via autocrine TNF- $\alpha$ signaling. Prostate, 77(8): 866-877.

Davie AH, Beltran H, Zoubeid A. (2018). Cellular plasticity and the neuroendocrine phenotype in prostate cancer. Nat Rev Urol, 15: 271-286.

Miao L, Yang L, Li R, Rodrigues DN, Crespo M, Hsieh JT, . . . Raj GV. (2017). Disrupting androgen receptor signaling induces snail-mediated epithelial-mesenchymal plasticity in prostate cancer. Cancer Res, 77(11): 3101-3112.

Quintanal-Villalonga Á, Chan JM, Yu HA, Pe'Er D, Sawyers CL, Sen T, Rudin CM. (2020). Lineage plasticity in cancer: a shared pathway of therapeutic resistance. Nat Rev Clin Oncol, 17(6):360-371.

Pal SK, Patel J, He M, Foulk B, Kraft K, Smirnov DA, . . Jones JO. (2018). Identification of mechanisms of resistance to treatment with abiraterone acetate or enzalutamide in patients with castration-resistant prostate cancer (CRPC). Cancer, 124(6):1216-1224. 
Song B, Park SH, Zhao JC, Fong KW, Li S, Lee Y, . . Yu J. (2019). Targeting FOXA1-mediated repression of TGF- $\beta$ signaling suppresses castration-resistant prostate cancer progression. J Clin Investig, 129(2): 569582.

Paller C, Pu H, Begemann DE, Wade CA, Hensley PJ, Kyprianou N. (2019). TGF- $\beta$ receptor I inhibitor enhances response to enzalutamide in a pre-clinical model of advanced prostate cancer. Prostate, 79(1): $31-43$.

Liu Q, Tong D, Liu G, Xu J, Do K, Geary K, . . . Jiang J. (2017). Metformin reverses prostate cancer resistance to enzalutamide by targeting TGF-31/STAT3 axis-regulated EMT. Cell Death Dis, 8(8): e3007.

Hensley PJ, Cao Z, Pu H, Dicke H. (2019). Predictive and targeting value of IGFBP-3 in therapeutically resistant prostate cancer. Am J Clin Exp Urol, 7(3): 188-202.

Ku SY, Rosario S, Wang Y, Mu P, Seshadri M. (2017). Rb1 and Trp53 cooperate to suppress prostate cancer lineage plasticity, metastasis, and antiandrogen resistance. Science, 355(6320): 78-83.

Ge R, Wang Z, Montironi R, Jiang Z, Cheng M, Santoni M, . . . Cheng L. (2020). Epigenetic modulations and lineage plasticity in advanced prostate cancer. Ann Oncol, 31(4): 470-479.

Xiao L, Tien JC, Vo J, Tan M, Parolia A, Zhang Y, . . . Chinnaiyan AM. (2018). Epigenetic reprogramming with antisense oligonucleotides enhances the effectiveness of androgen receptor inhibition in castration-resistant prostate cancer. Cancer Res, 78(20):5731-5740.

Carceles-Cordon M, Kelly WK, Gomella L, Knudsen KE, Rodriguez-Bravo V, Domingo-Domenech J. (2020). Cellular rewiring in lethal prostate cancer: the architect of drug resistance. Nat Rev Urol, 17(5): 292-307.

Zhang Y, Zheng D, Zhou T, Song H, Hulsurkar M, Su N, . . . Li W. (2018). Androgen deprivation promotes neuroendocrine differentiation and angiogenesis through CREB-EZH2-TSP1 pathway in prostate cancers. Nat Commun, 9(1): 4080.

Luo J, Wang K, Yeh S, Sun Y, Liang L, Xiao Y, . . . Chang C. (2019). LncRNA-p21 alters the antiandrogen enzalutamide-induced prostate cancer neuroendocrine differentiation via modulating the EZH2/STAT3 signaling. Nat Commun, 10(1): 2571.

Mu P, Zhang Z, Benelli M, Karthaus WR, Hoove E. (2017). SOX2 promotes lineage plasticity and antiandrogen resistance in TP53and RB1-deficient prostate cance. Science, 355: 84-88.

Metz EP, Wilder PJ, Dong J, Datta K, Rizzino A. (2020). Elevating SOX2 in prostate tumor cells upregulates expression of neuroendocrine genes, but does not reduce the inhibitory effects of enzalutamide. J Cell Physiol, 235(4): 3731-3740.

Nouri M, Massah S, Caradec J, Lubik AA, Li N, Truong S, . . . Buttyan R. (2020). Transient Sox9 Expression Facilitates Resistance to Androgen-Targeted Therapy in Prostate Cancer. Clin Cancer Res, 26(7): 1678-1689.

Flores-Morales A, Bergmann TB, Lavallee C, Batth TS, Lin D, Lerdrup M, . . . Iglesias-Gato D. (2019). Proteogenomic characterization of patient-derived xenografts highlights the role of REST in neuroendocrine differentiation of castration-resistant prostate cancer. Clin Cancer Res, 25(2): 595-608.

Li Y, Donmez N, Sahinalp C, Xie N, Wang Y, Xue H, . . . Dong X. (2017). SRRM4 drives neuroendocrine transdifferentiation of prostate adenocarcinoma under androgen receptor pathway inhibition. Eur Urol, 71(1): $68-78$.

Tiwari R, Manzar N, Bhatia V, Yadav A, Nengroo MA, Datta D, . . Ateeq B. (2020). Androgen deprivation upregulates SPINK1 expression and potentiates cellular plasticity in prostate cancer. Nat Commun, 11(1): 384. 
Dardenne E, Beltran H, Benelli M, Gayvert K, Berger A, Puca L, . . . Rickman DS. (2016). N-Myc induces an EZH2-mediated transcriptional program driving neuroendocrine prostate cancer. Cancer Cell, 30(4): 563-577.

Berger A, Brady NJ, Bareja R, Robinson B, Conteduca V, Augello MA, . . . Rickman DS. (2019). N-Mycmediated epigenetic reprogramming drives lineage plasticity in advanced prostate cancer. J Clin Invest, 130: 3924-3940.

Nerlakanti N, Yao J, Nguyen DT, Patel AK, Eroshkin AM, Lawrence HR, . . . Mahajan K. (2018). Targeting the BRD4-HOXB13 coregulated transcriptional networks with bromodomain-kinase inhibitors to suppress metastatic castration-resistant prostate cancer. Mol Cancer Ther, 17(12): 2796-2810.

Bishop JL, Thaper D, Vahid S, Davies A, Ketola K, Kuruma H, . . Z Zoubeidi A. (2017). The master neural transcription factor BRN2 is an androgen receptor-suppressed driver of neuroendocrine differentiation in prostate cancer. Cancer Discov, 7(1): 54-71.

Yasumizu Y, Rajabi H, Jin C, Hata T, Pitroda S, Long MD, . . . Kufe D. (2020). MUC1-C regulates lineage plasticity driving progression to neuroendocrine prostate cancer. Nat Commun, 11(1): 338.

Bhagirath D, Yang TL, Tabatabai ZL, Majid S, Dahiya R, Tanaka Y, Saini S. (2019). BRN4 is a novel driver of neuroendocrine differentiation in castration-resistant prostate cancer and is selectively released in extracellular vesicles with BRN2. Clin Cancer Res, 25(21): 6532-6545.

Reina-Campos M, Linares JF, Duran A, Cordes T, L'hermitte A, Badur MG, . . . Diaz-Meco MT. (2019). Increased serine and one-carbon pathway metabolism by PKC $\lambda / \iota$ deficiency promotes neuroendocrine prostate cancer. Cancer Cell, 35(3): 385-400.

Zhang Z, Zhou C, Li X, Barnes SD, Deng S, Hoover E, . . . Mu P. (2020). Loss of CHD1 promotes heterogeneous mechanisms of resistance to AR-targeted therapy via chromatin dysregulation. Cancer Cell, 37(4): 584-598.

Faugeroux V, Pailler E, Oulhen M, Deas O, Brulle-Soumare L, Hervieu C, . . . Farace F. (2020). Genetic characterization of a unique neuroendocrine transdifferentiation prostate circulating tumor cell-derived eXplant model. Nat Commun, 11(1): 1884.

Jones VS, Huang RY, Chen LP, Chen ZS, Fu L, Huang RP. (2016). Cytokines in cancer drug resistance: Cues to new therapeutic strategies. Biochim Biophys Acta, 1865(2): 255-265.

Culig Z, Puhr M. (2018). Interleukin-6 and prostate cancer: Current developments and unsolved questions. Mol Cell Endocrinol, 462(Pt A): 25-30.

Canesin G, Krzyzanowska A, Hellsten R, Bjartell A. (2020). Cytokines and Janus kinase/signal transducer and activator of transcription signaling in prostate cancer: overview and therapeutic opportunities. Curr Opin Endocr Metab Res, 10(6): 36-42.

Liu C, Zhu Y, Lou W, Cui Y, Evans CP, Gao AC. (2014). Inhibition of constitutively active Stat3 reverses enzalutamide resistance in LNCaP derivative prostate cancer cells. Prostate, 74(2): 201-209.

Wang C, Peng G, Huang H, Liu F, Kong DP, Dong KQ, . . . Sun YH. (2018). Blocking the Feedback loop between neuroendocrine differentiation and macrophages improves the therapeutic effects of enzalutamide (MDV3100) on prostate cancer. Clin Cancer Res, 24(3): 708-723.

Calcinotto A, Spataro C, Zagato E, Di Mitri D, Gil V, Crespo M, . . . Alimonti A. (2018). IL-23 secreted by myeloid cells drives castration-resistant prostate cancer. Nature, 559(7714): 363-369.

Jung Y, Cackowski FC, Yumoto K, Decker AM, Wang J, Kim JK, . . . Taichman RS. (2018). CXCL12 $\gamma$ promotes metastatic castration-resistant prostate cancer by inducing cancer stem cell and neuroendocrine phenotypes. Cancer Res, 78(8): 2026-2039. 
Li S, Fong KW, Gritsina G, Zhang A, Zhao JC, Kim J, . . . Yu J. (2019). Activation of MAPK signaling by CXCR7 leads to enzalutamide resistance in prostate cancer. Cancer Res, 79(10): 2580-2592.

Rafiei S, Gui B, Wu J, Liu XS, Kibel AS, Jia L. (2019). Targeting the MIF/CXCR7/AKT Signaling pathway in castration-resistant prostate cancer. Mol Cancer Res, 17(1): 263-276.

Luo Y, Azad AK, Karanika S, Basourakos SP, Zuo X, Wang J, . . . Thompson TC. (2018). Enzalutamide and CXCR7 inhibitor combination treatment suppresses cell growth and angiogenic signaling in castrationresistant prostate cancer models. Int J Cancer, 142(10): 2163-2174.

Pal SK, Moreira D, Won H, White SW, Duttagupta P, Lucia M, . . . Kortylewski M. (2019). Reduced T-cell numbers and elevated levels of immunomodulatory cytokines in metastatic prostate cancer patients de novo resistant to abiraterone and/or enzalutamide therapy. Int J Mol Sci, 20(8):1831.

Si W, Shen J, Zheng H, Fan W. (2019). The role and mechanisms of action of microRNAs in cancer drug resistance. Clin Epigenetics, 11(1):25.

Fletcher CE, Sulpice E, Combe S, Shibakawa A, Leach DA, Hamilton MP, . . . Bevan CL. (2019). Androgen receptor-modulatory microRNAs provide insight into therapy resistance and therapeutic targets in advanced prostate cancer. Oncogene, 38(28): 5700-5724.

Fernandes RC, Toubia J, Townley S, Hanson AR, Dredge BK, Pillman KA, . . . Selth LA. (2019). MicroRNA-194 promotes lineage plasticity in advanced prostate cancer. Retrieved from https://doi.org/10.1101/752709.

Lin SC, Kao CY, Lee HJ, Creighton CJ, Ittmann MM, Tsai SJ, . . . Tsai MJ. (2016). Dysregulation of miRNAs-COUP-TFII-FOXM1-CENPF axis contributes to the metastasis of prostate cancer. Nat Commun, 7: 11418.

Chen W, Yao G, Zhou K. (2019). miR-103a-2-5p/miR-30c-1-3p inhibits the progression of prostate cancer resistance to androgen ablation therapy via targeting androgen receptor variant 7. J Cell Biochem, 120(8): 14055-14064.

Ebron JS, Shankar E, Singh J, Sikand K, Weyman CM, Gupta S, . . Shukla GC. (2019). MiR-644a disrupts oncogenic transformation and warburg effect by direct modulation of multiple genes of tumor-promoting pathways. Cancer Res, 79(8): 1844-1856.

Aragon IM, Cendon Y, Lorente D, Mejorada RL. (2019). Implications of single nucleotide polymorphisms (SNPs) in androgen related-genes in outcome of metastatic castration-resistant prostate cancer (mCRPC) patients treated with abiraterone (Abi) and enzalutamide (Enza). Annals of Oncology, 30: 346.

Shiota M, Fujimoto N, Imada K, Yokomizo A, Itsumi M, Takeuchi A, . . . Naito S. (2016). Potential role for YB-1 in castration-resistant prostate cancer and resistance to enzalutamide through the androgen receptor V7. J Natl Cancer Inst, 108(7):10.

Shiota M, Fujimoto N, Itsumi M, Takeuchi A, Inokuchi J, Tatsugami K, . . . Eto M. (2017). Gene polymorphisms in antioxidant enzymes correlate with the efficacy of androgen-deprivation therapy for prostate cancer with implications of oxidative stress. Ann Oncol, 28(3): 569-575.

Fujimoto N, Shiota M, Tomisaki I, Minato A. (2017). Gene polymorphism-related individual and interracial differences in the outcomes of androgen deprivation therapy for prostate cancer. Clin Genitourin Cancer , $15(3): 337-342$.

Alizadeh M, Sazegar H, Zia N, Farsani FM. (2018). Study of the effects of rs137852595 single-nucleotide polymorphism on drug resistance of androgen receptor against Enzalutamide treatments in patients with prostate. Retrieved from https://www.researchgate.net/publication/332544381.

Kaviani B, Sazgar H, Zia Jahromi N, Mohamadi Farsani F. (2018). Investigation of drug resistance against treatment with Enzalutamide medicine in individuals diagnosed with prostate cancer and studying the 
effect of rs137852574 single-nucleotide polymorphism in drug resistance in the human population of Isfahan province. N Cell Mol Biotechnol J, 2018, 8(29): 69-78.

Farah E, Li C, Cheng L, Kong Y, Lanman NA, Pascuzzi P, . . . Liu X. (2019). NOTCH signaling is activated in and contributes to resistance in enzalutamide-resistant prostate cancer cells. J Biol Chem, 294(21): 8543-8554.

Kohrt SE, Awadallah WN, Phillips RA, Case TC, Jin R, Nanda JS, . . . Grabowska MM. (2020). Identification of genes required for enzalutamide resistance in castration-resistant prostate cancer cells. Retrieved from https://doi.org/10.1101/2020.03.27.011825.

Yuan M, Gao Y, Li L, Sun W, Cheng H, Li T, . . . Wu X. (2019). Phospholipase C (PLC)epsilon promotes androgen receptor antagonist resistance via the bone morphogenetic protein (BMP)-6/SMAD axis in a castration-resistant prostate cancer cell line. Med Sci Monit, 25: 4438-4449.

Kong Y, Cheng L, Mao F, Zhang Z, Zhang Y, Farah E, . . . Liu X. (2018). Inhibition of cholesterol biosynthesis overcomes enzalutamide resistance in castration-resistant prostate cancer (CRPC). J Biol Chem, 293(37): 14328-14341.

Neuwirt H, Bouchal J, Kharaishvili G, Ploner C, Jöhrer K, Pitterl F, . . Eder IE. (2020). Cancer-associated fibroblasts promote prostate tumor growth and progression through upregulation of cholesterol and steroid biosynthesis. Cell Commun Signal, 18(1):11.

Buttigliero C, Tucci M, Bertaglia V, Vignani F, Bironzo P, Di Maio M, Scagliotti GV. (2015). Understanding and overcoming the mechanisms of primary and acquired resistance to abiraterone and enzalutamide in castration resistant prostate cancer. Cancer Treat Rev, 41(10): 884-892.

Xu L, Chen J, Liu W, Liang C, Hu H, Huang J. (2019). Targeting androgen receptor-independent pathways in therapy-resistant prostate cancer. Asian J Urol, 6(1): 91-98.

Lawrence MG, Obinata D, Sandhu S, Selth LA, Wong SQ, Porter LH, . . Risbridger GP. (2018). Patientderived models of abiraterone- and enzalutamide-resistant prostate cancer reveal sensitivity to ribosomedirected therapy. Eur Urol, 74(5): 562-572.

Gui B, Gui F, Takai T, Feng C, Bai X, Fazli L, . . Jia L. (2019). Selective targeting of PARP-2 inhibits androgen receptor signaling and prostate cancer growth through disruption of FOXA1 function. Proc Natl Acad Sci U S A, 116(29): 14573-14582. 


\begin{tabular}{|c|c|c|c|}
\hline Mechanisms & $\begin{array}{l}\text { Signalling } \\
\text { pathways/Targets }\end{array}$ & Solutions & References \\
\hline \multirow[t]{11}{*}{ AR reactivation } & $\begin{array}{l}\text { AR enhancer }(650 \mathrm{~kb} \\
\text { centromeric) }\end{array}$ & & $\begin{array}{l}\text { (Takeda, Spisák et al., } \\
\text { 2018) }\end{array}$ \\
\hline & Galectin-3 & & $\begin{array}{l}\text { (Dondoo, Fukumori et al., } \\
\text { 2017) }\end{array}$ \\
\hline & BMI1 & PTC209 (BMI1 inhibitor) & (Zhu, Zhao et al., 2018) \\
\hline & FOXA1/CREB5/AR & & (Hwang, Seo et al., 2019) \\
\hline & $\begin{array}{l}\text { IKBKE/Hippo/c- } \\
\text { Myc/AR }\end{array}$ & & $\begin{array}{l}\text { (Bainbridge, Walker et al., } \\
\text { 2020) }\end{array}$ \\
\hline & EphB4/c-Myc/AR & EphB4 inhibitors & (Li, Lanman et al., 2020) \\
\hline & elF4F & & (Liu, Horn et al., 2019) \\
\hline & STAT3/AR & GPA500 & (Thaper, Vahid et al., 2018) \\
\hline & AR/AR-Vs & Triptolide & (Han, Huang et al., 2017) \\
\hline & $\mathrm{AR}$ & Tetraaryl cyclobutane (CBs) & $\begin{array}{l}\text { (Pollock, Wardell et al., } \\
\text { 2016) }\end{array}$ \\
\hline & $\mathrm{AR}$ & ARCC-4/UT-34/IRF8 & (Salami, Alabi et al., 2018) \\
\hline \multirow[t]{11}{*}{ AR splice variants } & IncRNA Malat1 & & $\begin{array}{l}\text { (Tummala, Lou et al., 2017; } \\
\text { Stone, 2017; Zhao, Wang et } \\
\text { al., 2018) }\end{array}$ \\
\hline & $\begin{array}{l}\text { ACK1/pY88- } \\
\text { H4/WDR5/MLL2/AR, } \\
\text { AR-V7 }\end{array}$ & & $\begin{array}{l}\text { (Mahajan, Malla et al., } \\
\text { 2017) }\end{array}$ \\
\hline & AR-V7 & SPT & (Lam, Nguyen et al., 2020) \\
\hline & AVPR1A & & (Zhao, Peacock et al., 2019) \\
\hline & $\begin{array}{l}\text { NK cells/microRNA-34, } \\
\text { microRNA-449/AR-V7, } \\
\text { EZH2 }\end{array}$ & & (Lin, Chou et al., 2017) \\
\hline & HSP70/STUB1/AR-V7 & & (Liu, Lou et al., 2018) \\
\hline & AKR1C3/AR-V7 & & (Liu, Yang et al., 2019) \\
\hline & AR-V7 & $\begin{array}{l}\text { ABT263 (BCL2 protein } \\
\text { inhibitor)/IPI-9119 (fatty acid } \\
\text { synthase } \\
\text { inhibitor)/Luteolin/CDDO- } \\
\text { Me/Degarelix }\end{array}$ & $\begin{array}{l}\text { (Zadra, Ribeiro et al., 2019; } \\
\text { Naiki-Ito, Naiki et al., } \\
\text { 2019; Khurana, Chandra et } \\
\text { al., 2020; Cucchiara, Yang } \\
\text { et al., 2019) }\end{array}$ \\
\hline & c-Myc/AR-Vs, AR-FL & Quiflapon (5-Lox inhibitor) & $\begin{array}{l}\text { (BaiCao et al., 2019; } \\
\text { Monga, Subramani et al., } \\
\text { 2020) }\end{array}$ \\
\hline & GATA2/AR-FL, AR-Vs & & $\begin{array}{l}\text { (Chaytor, Simcock et al., } \\
\text { 2019) }\end{array}$ \\
\hline & BRD4 & $\begin{array}{l}\text { JQI//-BET762 } \\
\text { inhibitors) }\end{array}$ & $\begin{array}{l}\text { (Asangani, Dommeti et al., } \\
\text { 2014; Chan, Selth et al., } \\
\text { 2015) }\end{array}$ \\
\hline
\end{tabular}




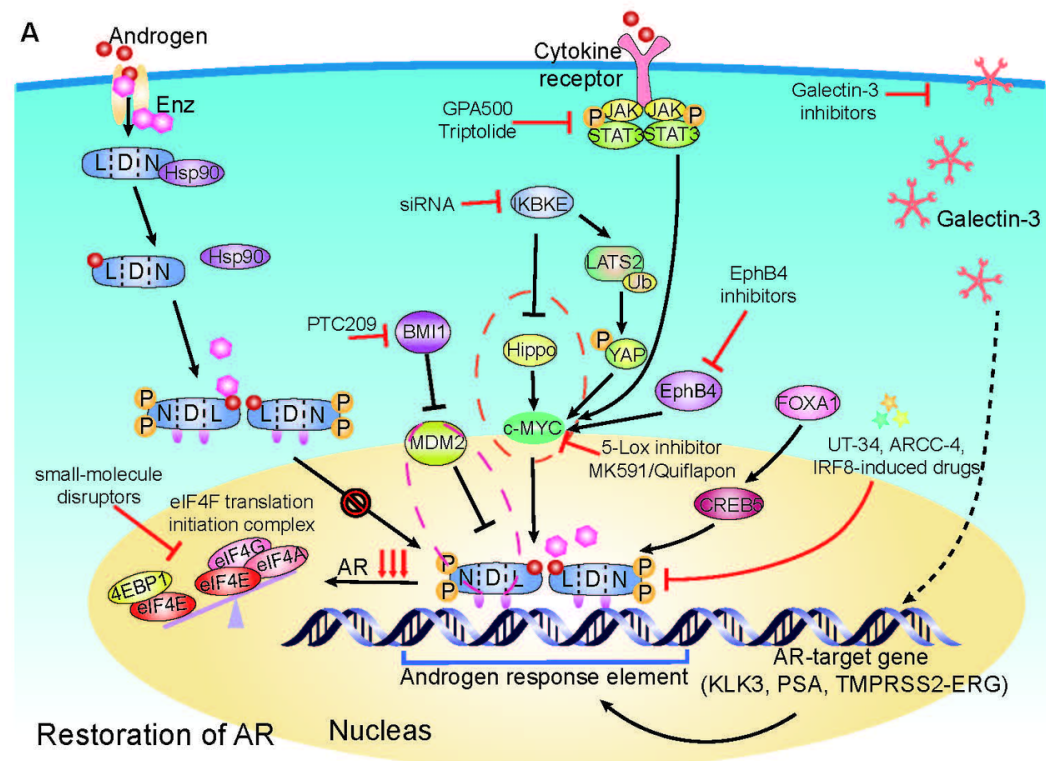

B

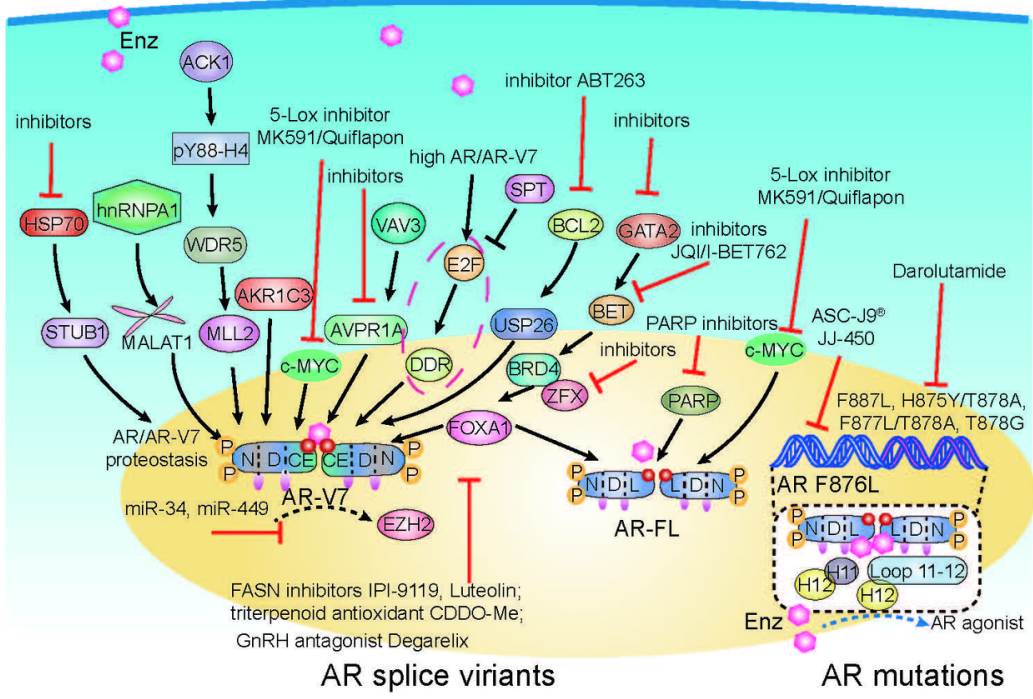




\section{ब6}
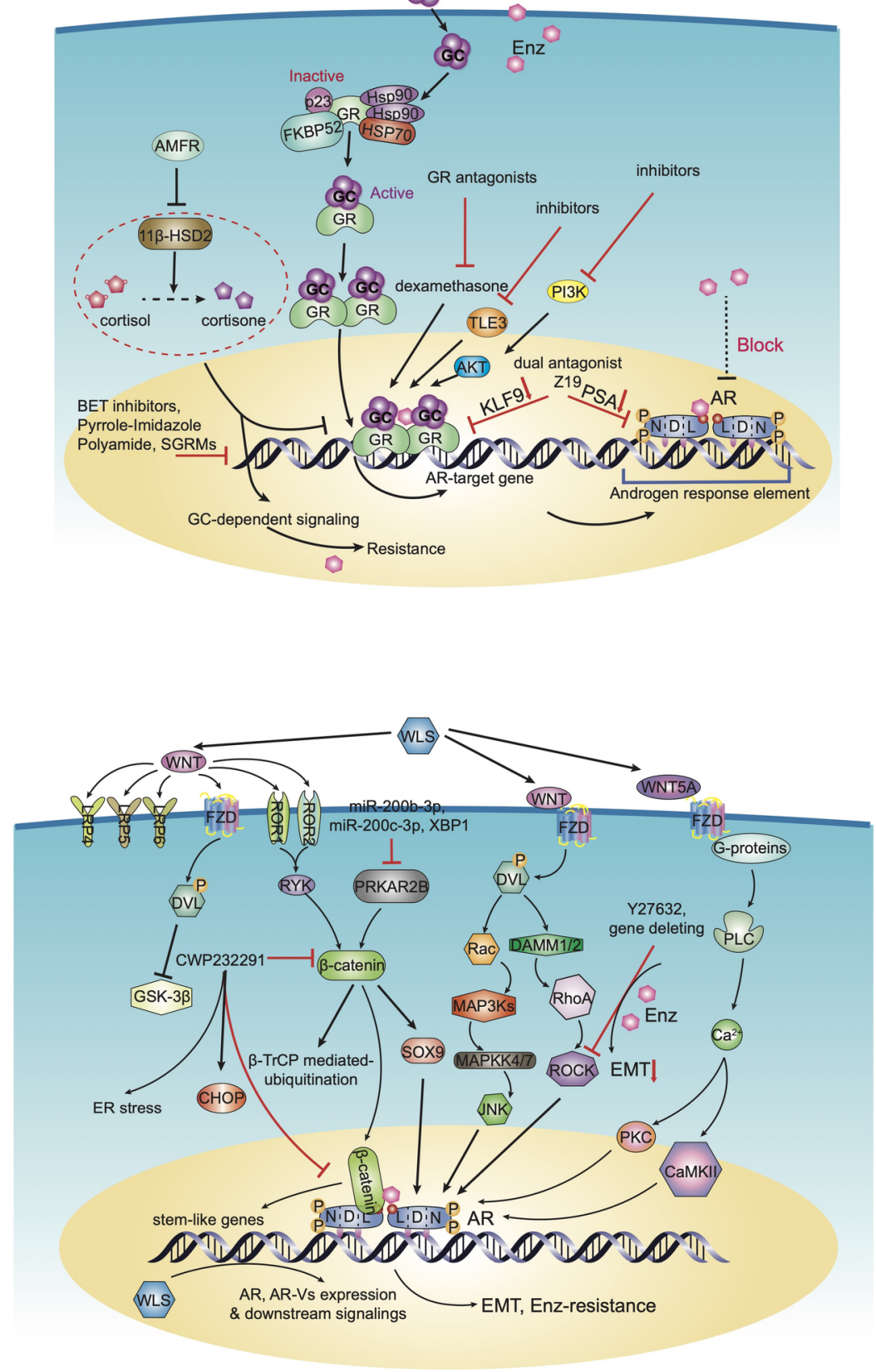


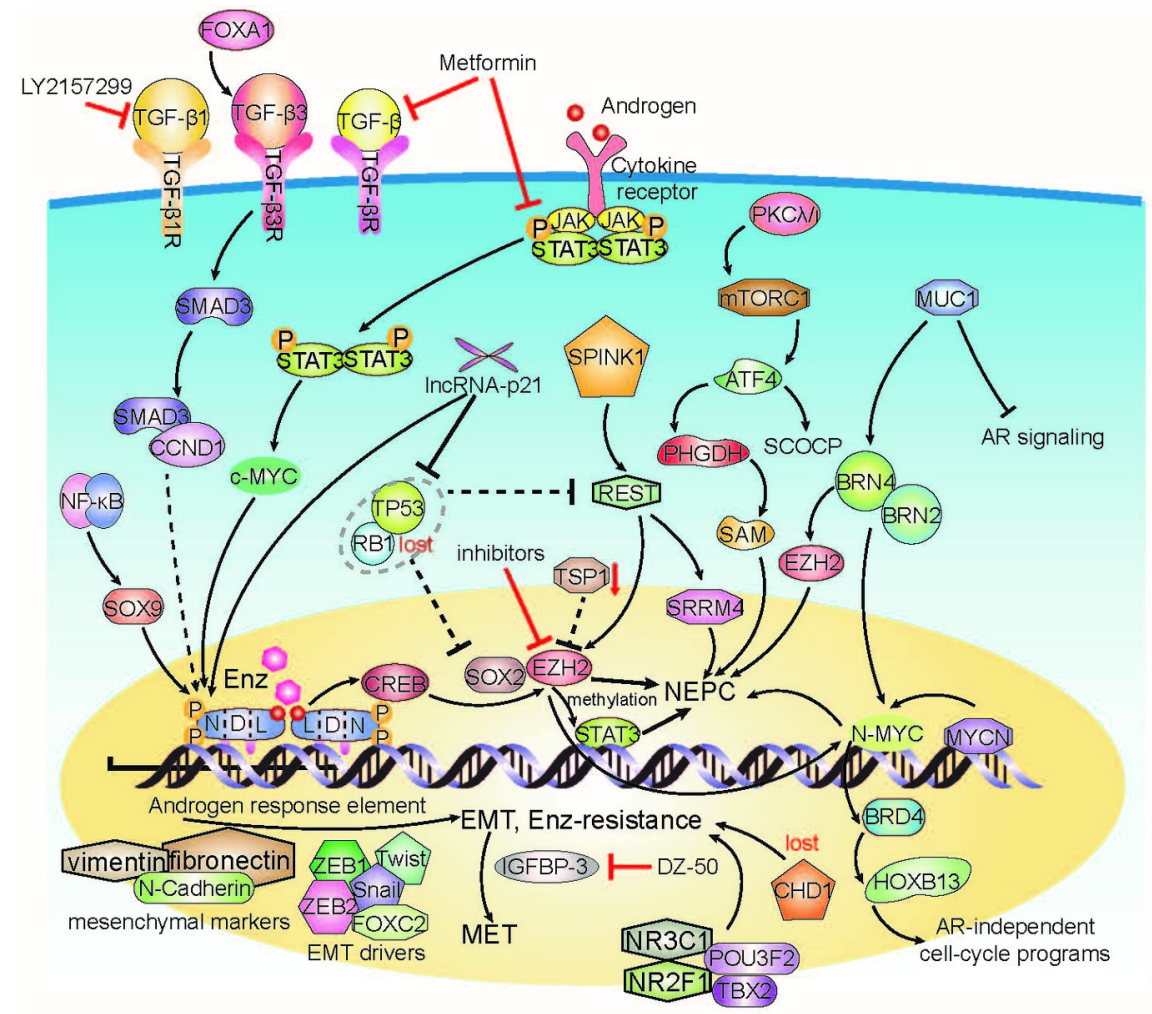

Lineage plasticity and phenotype switching 